Supporting information and Spectral Data for:

\title{
Copper(II)-catalyzed Aminohydroxylation of Olefins
}

\author{
David J. Michaelis, Christopher J. Shaffer, Tehshik P. Yoon* \\ Department of Chemistry, University of Wisconsin-Madison, 1101 University Avenue, Madison, \\ Wisconsin 53706-1396
}

\section{General Information.}

All commercially available styrene derivatives were freshly distilled before use except 2-vinylnaphthalene, which was purified by flash chromatography on silica gel with $3 \%$ EtOAc in hexanes as eluent. HMPA was distilled before use and stored over $4 \AA$ molecular sieves. $\mathrm{CH}_{2} \mathrm{Cl}_{2}$ was purified by distillation from $\mathrm{CaH}_{2}$ immediately prior to use. trans-(2-Phenylsulfonyl)-3-phenyloxaziridine was synthesized as previously described. ${ }^{1}$ All other compounds were purchased from Aldrich or Strem and used without further purification. Flash chromatography was performed with Purasil $60 \AA$ silica gel (230-400 mesh). All glassware was oven-dried for at least 1 hour before use. Diastereomeric ratios for all compounds were determined by ${ }^{1} \mathrm{H}$ NMR analysis of the crude reaction mixtures.

${ }^{1} \mathrm{H}$ and ${ }^{13} \mathrm{C}$ NMR data were obtained using Varian Inova-500 (NSF CHE-9629688) and Varian Unity-500 (NSF CHE-8813550, NIH 1 S10 RR0 4981-10, NSF CHE-9629688) spectrometers. All ${ }^{1} \mathrm{H}$ and ${ }^{13} \mathrm{C}$ spectra were referenced to TMS $(0.00 \mathrm{ppm})$ or DMSO $(2.5 \mathrm{ppm})$, and $\mathrm{CDCl}_{3}(77.23 \mathrm{ppm})$ or DMSO (39.5 ppm) respectively. IR spectral data were obtained using a Bruker Vector 22 spectrometer (thin film on $\mathrm{NaCl}$ ). Melting points were obtained using a Mel-Temp II (Laboratory Devices, Inc., USA) melting point apparatus. Mass spectrometry was performed with a Micromass LCT (electrospray ionization, time-of-flight analyzer). These facilities are supported by NSF grant \# CHE-9709005 and the University of Wisconsin.

General procedure for olefin aminohydroxylation: To a dry 2 dram vial containing a stir bar was added $8 \mathrm{mg}$ (.027 mmol) copper(II) trifluoroacetate hydrate, followed by $470 \mathrm{mg}$ (1.8 mmol) trans-(2-phenylsulfonyl)-3phenyloxaziridine (1) and $1 \mathrm{~mL}$ freshly distilled $\mathrm{CH}_{2} \mathrm{Cl}_{2}$. A second vial was charged with the appropriate olefin (1.2 mmol), $21 \mu \mathrm{L}$ HMPA $(120 \mu \mathrm{mol})$ and $1 \mathrm{~mL} \mathrm{CH}_{2} \mathrm{Cl}_{2}$. The olefin solution was then added via syringe to the oxaziridine solution, and the empty vial rinsed with two portions of $0.2 \mathrm{ml} \mathrm{CH}_{2} \mathrm{Cl}_{2}$. The vial was sealed with a septum, and the reaction was stirred under $\mathrm{N}_{2}$ until completion. The reaction mixture was then diluted with $2 \mathrm{~mL}$ $\mathrm{CH}_{2} \mathrm{Cl}_{2}$ and $2 \mathrm{~mL}$ hexanes, and this mixture was directly loaded onto a silica gel column and purified by chromatography to give the aminal product as a mixture of two diastereomers.

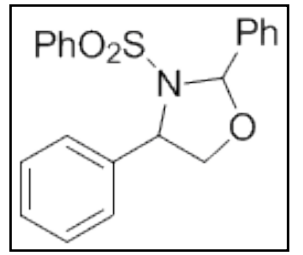

$\boldsymbol{N}$-Benzensulfonyl-2,4-diphenyl-1,3-oxazolane (Table 2, entry 1). Prepared according to the general procedure using $126 \mathrm{mg}$ styrene. The imine resulting from reduction of Davis's oxaziridine was inseparable from the product by flash chromatography (6\% to $9 \%$ EtOAc in hexanes as eluent). The clean product was obtained by treatment of the purified product and

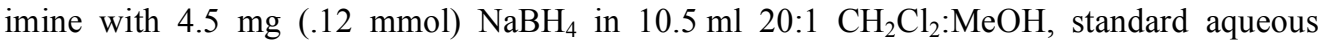
workup with sat. $\mathrm{NaHCO}_{3}$, and chromatographic purification using 1:6 EtOAc:hexanes as eluent. Yield 1: $376 \mathrm{mg}$ (1.03 mmol, 86\% yield), 1.6:1 cis:trans; yield 2: $381 \mathrm{mg}$ (1.04 mmol, $87 \%$ yield), 1.8:1 cis:trans. Major diastereomer $\left(2 S^{*}, 4 S^{*}\right)$ : IR (neat) $3063,3032,2878,1355,1167 ;{ }^{1} \mathrm{H}$ NMR $(500$ $\left.\mathrm{MHz}, \mathrm{CDCl}_{3}\right) \delta 7.76\left(\mathrm{~d}, 8.6 \mathrm{~Hz}, 2 \mathrm{H},-\mathrm{SO}_{2} \mathrm{PhH}\right), 7.56-7.62(\mathrm{~m}, 3 \mathrm{H}, \mathrm{ArH}), 7.45(\mathrm{t}, \mathrm{J}=8.3 \mathrm{~Hz}, 2 \mathrm{H}, \mathrm{ArH}), 7.32-7.40$ $(\mathrm{m}, 3 \mathrm{H}, \mathrm{ArH}), 7.21-7.30(\mathrm{~m}, 5 \mathrm{H}, \mathrm{Ar}-\mathrm{H}), 6.36\left(\mathrm{~s}, 1 \mathrm{H}, \mathbf{H}_{\text {aminal }}\right), 4.88\left(\mathrm{t}, \mathrm{J}=7.0 \mathrm{~Hz}, 1 \mathrm{H}, \mathrm{CH}-\mathrm{CH}_{2}\right), 4.19$ (dd, J = 9.1, $\left.7.2 \mathrm{~Hz}, 1 \mathrm{H}, \mathrm{CH}_{2}\right), 3.91\left(\mathrm{dd}, \mathrm{J}=9.1,7.0 \mathrm{~Hz}, 1 \mathrm{H}, \mathrm{CH}_{2}\right) ;{ }^{13} \mathrm{C}$ NMR $\left(500 \mathrm{MHz}, \mathrm{CDCl}_{3}\right): 138.6,138.1,137.7,133.5$, $129.4,129.1,128.8,128.7,128.3,128.2,127.4(0), 127.3(8), 92.7,73.4,63.4 ;$ HRMS $\left(\mathrm{ESI}^{+}\right)$calc'd for $\left[\mathrm{C}_{21} \mathrm{H}_{19} \mathrm{NO}_{3} \mathrm{SNa}\right]^{+}$requires $\mathrm{m} / z 388.0983$, found $\mathrm{m} / \mathrm{z} 388.0998$. Isolated as a white solid $\left(\mathrm{mp}=144-145^{\circ} \mathrm{C}\right)$. Minor diastereomer $\left(2 R^{*}, 4 S^{*}\right)$ : IR (neat) $3063,3032,2855,1350,1164 ;{ }^{1} \mathrm{H}$ NMR $\left(500 \mathrm{MHz}, \mathrm{CDCl}_{3}\right) \delta 7.52(\mathrm{~m}, 2 \mathrm{H}$, $\left.\mathrm{SO}_{2} \mathrm{PhH}\right), 7.10-7.40(\mathrm{~m}, 13 \mathrm{H}, \mathrm{ArH}), 6.36\left(\mathrm{~s}, 1 \mathrm{H}, \mathbf{H}_{\text {aminal }}\right), 5.10\left(\mathrm{dd}, \mathrm{J}=6.3,2.0 \mathrm{~Hz}, 1 \mathrm{H}, \mathrm{CH}_{-} \mathrm{CH}_{2}\right), 4.39$ (dd, J = 8.9, $\left.6.3 \mathrm{~Hz}, 1 \mathrm{H}, \mathrm{CH}_{2}\right), 3.98\left(\mathrm{dd}, \mathrm{J}=8.9,2.0 \mathrm{~Hz}, 1 \mathrm{H}, \mathrm{CH}_{2}\right) ;{ }^{13} \mathrm{C} \mathrm{NMR}\left(500 \mathrm{MHz}, \mathrm{CDCl}_{3}\right) \delta 140.5,138.7,138.5,132.1$ $129.3128 .7128 .5,128.5,128.3128 .0,127.6$ 127.0, 92.4, 73.1, 63.0; HRMS (EI ${ }^{+}$) calc'd for $\left[\mathrm{C}_{21} \mathrm{H}_{19} \mathrm{NO}_{3} \mathrm{SH}^{+}\right.$ requires $\mathrm{m} / \mathrm{z}$ 364.1007, found $\mathrm{m} / \mathrm{z}$ 364.1022. Isolated as a clear oil. Alternative Additives (DMPU and Pyridine): Styrene was subjected to the conditions of the general procedure except 1,3-Dimethyl-3,4,5,6-tetrahydro-2(1H)pyrimidinone (DMPU) $(15 \mathrm{mg}, .12 \mathrm{mmol})$ or pyridine $(9.5 \mathrm{mg}, .12 \mathrm{mmol})$ was substituted for HMPA. The reaction 
containing DMPU was complete in $12 \mathrm{~h}$ and the reaction containing pyridine complete at $16 \mathrm{~h}$. Yield using DMPU: $361 \mathrm{mg}$ (.987 mmol, 81.6\%), 3.3:1 cis:trans. Yield using pyridine: $394 \mathrm{mg}$ (1.08 mmol, 89.4\%), 2.3:1 cis:trans.

$\mathrm{N}$-Benzensulfonyl-4-(4-trifluoromethylphenyl)-2-phenyl-1,3-oxazolane (Table 2, entry 2). Prepared according to the general procedure using $207 \mathrm{mg}$ 4-trifluoromethylstyrene and purified on silica gel using 1:1 $\mathrm{CH}_{2} \mathrm{Cl}_{2}$ :hexanes as eluent. Yield 1: $388 \mathrm{mg}$ (.895 mmol, 74.4\% yield), 1.2:1 cis:trans; yield 2: $369 \mathrm{mg}$ (.852 mmol, 71.1\% yield), 1.2:1

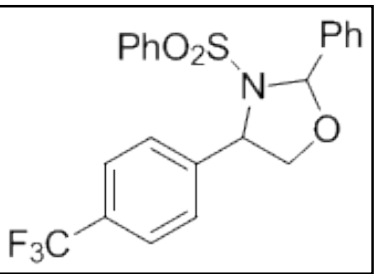
cis:trans. Major diastereomer $\left(2 S^{*}, 4 S^{*}\right)$ : IR (neat) $3065,2924,1326,1166 ;{ }^{1} \mathrm{H}$ NMR $\left(500 \mathrm{MHz}, \mathrm{CDCl}_{3}\right) \delta 7.79\left(\mathrm{dd}, 8.1,1.2 \mathrm{~Hz}, 2 \mathrm{H},-\mathrm{SO}_{2} \mathrm{PhH}\right), 7.58-7.68(\mathrm{~m}, 3 \mathrm{H}, \mathrm{ArH})$, 7.50-7.57 (m, 4H, ArH), 7.35-7.45 (m, 5H, ArH), 6.40 (s, 1H, $\left.\mathbf{H}_{\text {aminal }}\right), 4.95(\mathrm{t}, \mathrm{J}=6.9$ $\left.\mathrm{Hz}, 1 \mathrm{H}, \mathrm{CH}-\mathrm{CH}_{2}\right), 4.24\left(\mathrm{dd}, \mathrm{J}=9.0,6.9 \mathrm{~Hz}, 1 \mathrm{H}, \mathrm{CH}_{2}\right), 3.90(\mathrm{dd}, \mathrm{J}=6.9,9.0 \mathrm{~Hz}, 1 \mathrm{H}$, $\left.\mathrm{CH}_{2}\right) ;{ }^{13} \mathrm{C}$ NMR $\left(500 \mathrm{MHz}, \mathrm{CDCl}_{3}\right)$ 142.8, 137.7, 137.3, 133.7, 130.4, 129.5, 129.2, $128.8,128.2,127.7,127.2,125.8,124.2,92.8,73.1,62.9$; HRMS $\left(\mathrm{ESI}^{+}\right)$calc'd for $\left[\mathrm{C}_{22} \mathrm{H}_{18} \mathrm{~F}_{3} \mathrm{NO}_{3} \mathrm{SNa}\right]^{+}$requires $\mathrm{m} / \mathrm{z} 456.0857$, found $\mathrm{m} / \mathrm{z} 456.0874$. Isolated as a white solid $\left(\mathrm{mp}=88-91^{\circ} \mathrm{C}\right)$. Minor diastereomer $\left(2 R^{*}, 4 S^{*}\right)$ : IR (neat) $3068,2923,1326$, $1165 ;{ }^{1} \mathrm{H}$ NMR $\left(500 \mathrm{MHz}, \mathrm{CDCl}_{3}\right) \delta 7.52\left(\mathrm{~m}, 2 \mathrm{H},-\mathrm{SO}_{2} \mathrm{PhH}\right), 7.33-7.42(\mathrm{~m}, 8 \mathrm{H}, \mathrm{ArH}), 7.10-7.21(\mathrm{~m}, 4 \mathrm{H}, \mathrm{ArH})$, $6.42\left(\mathrm{~s}, 1 \mathrm{H}, \mathbf{H}_{\text {aminal }}\right), 5.13\left(\mathrm{dd}, \mathrm{J}=6.3,1.7 \mathrm{~Hz}, 1 \mathrm{H}, \mathbf{C H}-\mathrm{CH}_{2}\right), 4.41\left(\mathrm{dd}, \mathrm{J}=6.3,9.1 \mathrm{~Hz}, 1 \mathrm{H}, \mathrm{CH}_{2}\right), 3.96(\mathrm{dd}, \mathrm{J}=9.1$, $\left.1.7 \mathrm{~Hz}, 1 \mathrm{H}, \mathrm{CH}_{2}\right) ;{ }^{13} \mathrm{C}$ NMR $\left(500 \mathrm{MHz}, \mathrm{CDCl}_{3}\right)$ 142.4, 140.5, 138.1, 132.4, 130.5, 129.5, 128.6(3), 128.6(0), 128.3, 127.5, 126.8, 125.6, 124.1, 92.6, 72.8, 62.3; HRMS $\left(\mathrm{ESI}^{+}\right)$calc'd for $\left[\mathrm{C}_{22} \mathrm{H}_{18} \mathrm{~F}_{3} \mathrm{NO}_{3} \mathrm{SNa}\right]^{+}$requires $\mathrm{m} / \mathrm{z} 456.0857$, found $\mathrm{m} / \mathrm{z} 456.0871$. Isolated as a white solid $\left(\mathrm{mp}=161-162{ }^{\circ} \mathrm{C}\right)$.

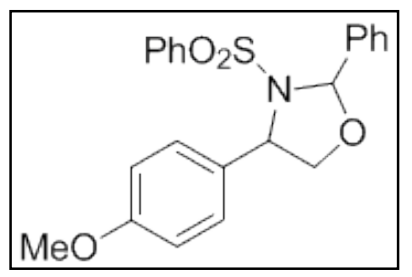

$N$-Benzensulfonyl-4-(4-methoxyphenyl)-2-phenyl-1,3-oxazolane (Table 2, entry 3). Prepared according to the general procedure using $161 \mathrm{mg} 4$-methoxystyrene and purified on silica gel using 3:1 $\mathrm{CH}_{2} \mathrm{Cl}_{2}$ :hexanes as eluent. Yield 1: $402 \mathrm{mg}(1.02$ mmol, $85.2 \%$ yield), 1.2:1 cis:trans; yield 2: $392 \mathrm{mg}$ (.992 mmol, 82.3\% yield), 1.4:1 cis:trans. Major diastereomer $\left(2 S^{*}, 4 S^{*}\right)$ : IR (neat) $3063,3003,2925,1354,1166 ;{ }^{1} \mathrm{H}$ NMR $\left(500 \mathrm{MHz}, \mathrm{CDCl}_{3}\right) \delta 7.76\left(\mathrm{~d}, \mathrm{~J}=7.0 \mathrm{~Hz}, 2 \mathrm{H},-\mathrm{SO}_{2} \mathrm{PhH}\right), 7.55-7.63(\mathrm{~m}, 3 \mathrm{H}$, $\operatorname{ArH}), 7.48(\mathrm{t}, \mathrm{J}=7.9 \mathrm{~Hz}, 2 \mathrm{H}, \operatorname{ArH}), 7.32-7.41(\mathrm{~m}, 3 \mathrm{H}, \operatorname{ArH}), 7.15(\mathrm{~d}, \mathrm{~J}=8.7,2 \mathrm{H}, 4-$ $\mathrm{OMePhH}), 6.79(\mathrm{~d}, \mathrm{~J}=8.7,2 \mathrm{H}, 4-\mathrm{OMePhH}), 6.34\left(\mathrm{~s}, 1 \mathrm{H}, \mathbf{H}_{\text {aminal }}\right), 4.83\left(\mathrm{t}, \mathrm{J}=6.9 \mathrm{~Hz}, 1 \mathrm{H}, \mathrm{CH}_{-} \mathrm{CH}_{2}\right), 4.14(\mathrm{dd}, \mathrm{J}=$ 9.0, 7.0 Hz, $\left.1 \mathrm{H}, \mathrm{CH}_{2}\right), 3.88\left(\mathrm{dd}, \mathrm{J}=9.0,6.9 \mathrm{~Hz}, 1 \mathrm{H}, \mathrm{CH}_{2}\right), 3.77\left(\mathrm{~s}, 3 \mathrm{H}, \mathrm{OCH}_{3}\right) ;{ }^{13} \mathrm{C} \mathrm{NMR}\left(500 \mathrm{MHz}, \mathrm{CDCl}_{3}\right) 159.5$,

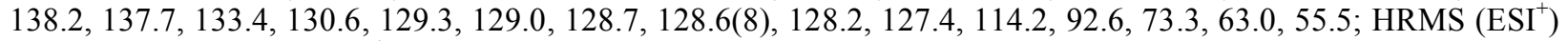
calc'd for $\left[\mathrm{C}_{22} \mathrm{H}_{21} \mathrm{NO}_{4} \mathrm{SNa}\right]^{+}$requires $\mathrm{m} / z$ 418.1089, found $\mathrm{m} / \mathrm{z} 418.1094$. Isolated as a white solid $\left(\mathrm{mp}=97-98{ }^{\circ} \mathrm{C}\right)$. Minor diastereomer $\left(2 R^{*}, 4 S^{*}\right)$ : IR (neat) $3063,2924,2852,1349,1163 ;{ }^{1} \mathrm{H}$ NMR $\left(500 \mathrm{MHz}, \mathrm{CDCl}_{3}\right) \delta 7.51-7.57$ $\left(\mathrm{m}, 2 \mathrm{H},-\mathrm{SO}_{2} \mathrm{PhH}\right), 7.34-7.43(\mathrm{~m}, 4 \mathrm{H}, \mathrm{ArH}), 7.12-7.23(\mathrm{~m}, 6 \mathrm{H}, \mathrm{ArH}), 6.66(\mathrm{~d}, \mathrm{~J}=8.6 \mathrm{~Hz}, 2 \mathrm{H}, 4-\mathrm{OMePhH}), 6.35$ $\left(\mathrm{s}, 1 \mathrm{H}, \mathbf{H}_{\text {aminal }}\right), 5.05\left(\mathrm{dd}, \mathrm{J}=6.4,2.0 \mathrm{~Hz}, 1 \mathrm{H}, \mathrm{CH}-\mathrm{CH}_{2}\right), 4.36\left(\mathrm{dd}, \mathrm{J}=8.9,6.4 \mathrm{~Hz}, 1 \mathrm{H}, \mathrm{CH}_{2}\right), 3.97(\mathrm{dd}, \mathrm{J}=8.9,2.0$ $\left.\mathrm{Hz}, 1 \mathrm{H}, \mathrm{CH}_{2}\right), 3.79\left(\mathrm{~s}, 3 \mathrm{H}, \mathrm{OCH}_{3}\right) ;{ }^{13} \mathrm{C}$ NMR $\left(500 \mathrm{MHz}, \mathrm{CDCl}_{3}\right) 159.7,140.8,138.7,132.0,130.7,129.5,129.3$, 128.6, 128.5, 127.6, 127.1, 114.0, 92.3, 73.1, 62.5, 55.5; HRMS $\left(\mathrm{ESI}^{+}\right)$calc'd for $\left[\mathrm{C}_{22} \mathrm{H}_{21} \mathrm{NO}_{4} \mathrm{SNa}\right]^{+}$requires $\mathrm{m} / z$ 418.1089 , found $m / z 418.1090$. Isolated as a white solid $\left(\mathrm{mp}=99-103{ }^{\circ} \mathrm{C}\right)$.

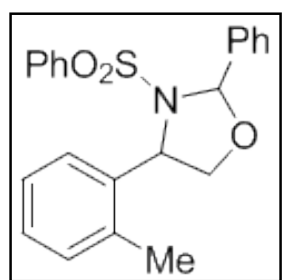

$N$-Benzensulfonyl-4-(2-methylphenyl)-2-phenyl-1,3-oxazolane (Table 2, entry 4). Prepared according to the general procedure using $141 \mathrm{mg}$ 2-methylstyrene. Yield 1: $381 \mathrm{mg}(1.0$ mmol, $84.3 \%$ yield), $1.0: 1$ cis:trans; yield $2: 384 \mathrm{mg}$ (1.01 mmol, $84.1 \%$ yield), 1.0:1 cis:trans. Diastereomer $1\left(2 R^{*}, 4 S^{*}\right)$ : IR (neat) $3065,3031,2949,1885,1350,1164 ;{ }^{1} \mathrm{H}$ NMR $\left(500 \mathrm{MHz}, \mathrm{CDCl}_{3}\right) \delta 7.52\left(\mathrm{~m}, 2 \mathrm{H}, \mathrm{SO}_{2} \mathrm{PhH}\right), 7.31-7.41(\mathrm{~m}, 4 \mathrm{H}, \mathrm{ArH}), 7.19(\mathrm{~d}, \mathrm{~J}=8.6 \mathrm{~Hz}, 2 \mathrm{H}$, $\operatorname{ArH}), 7.04-7.21(\mathrm{~m}, 5 \mathrm{H}, \operatorname{ArH}), 6.82(\mathrm{t}, \mathrm{J}=8.0,1 \mathrm{H}, \operatorname{ArH}), 6.42\left(\mathrm{~s}, 1 \mathrm{H}, \mathbf{H}_{\text {aminal }}\right), 5.41(\mathrm{dd}, \mathrm{J}=$ 6.6, $\left.2.5 \mathrm{~Hz}, 1 \mathrm{H}, \mathrm{CH}-\mathrm{CH}_{2}\right), 4.40\left(\mathrm{dd}, \mathrm{J}=8.8,6.6 \mathrm{~Hz}, 1 \mathrm{H}, \mathrm{CH}_{2}\right), 3.92(\mathrm{dd}, \mathrm{J}=8.8,2.5 \mathrm{~Hz}, 1 \mathrm{H}$, $\left.\mathrm{CH}_{2}\right), 2.47\left(\mathrm{~s}, 3 \mathrm{H}, \mathrm{CH}_{3}\right) ;{ }^{13} \mathrm{C}$ NMR $\left(500 \mathrm{MHz}, \mathrm{CDCl}_{3}\right)$ 143.2, 141.0, 139.2, 138.5, 134.6, 133.1, 131.8, 131.1, 131.0, 130.5, 130.0, 129.9, 129.5, 129.2, 95.2, 75.4, 61.1, 22.2; HRMS (ESI ${ }^{+}$) calc'd for $\left[\mathrm{C}_{22} \mathrm{H}_{21} \mathrm{NO}_{3} \mathrm{SNa}\right]^{+}$requires $\mathrm{m} / \mathrm{z} 402.1140$, found $\mathrm{m} / \mathrm{z} 402.1160$. Isolated as a colorless oil. Diastereomer 2 $\left(2 S^{*}, 4 S^{*}\right)$ : IR (neat) $3064,3031,2946,2880,1356,1168 ;{ }^{1} \mathrm{H} \mathrm{NMR}\left(500 \mathrm{MHz}, \mathrm{CDCl}_{3}\right) \delta 7.75(\mathrm{~d}, \mathrm{~J}=7.3 \mathrm{~Hz}, 2 \mathrm{H}$, $\operatorname{ArH}), 7.68(\mathrm{~d}, \mathrm{~J}=7.7 \mathrm{~Hz}, 2 \mathrm{H}, \operatorname{ArH}), 7.58(\mathrm{t}, \mathrm{J}=7.7 \mathrm{~Hz}, 1 \mathrm{H}, \operatorname{ArH}), 7.35-7.50(\mathrm{~m}, 5 \mathrm{H}, \operatorname{ArH}), 7.25(\mathrm{~d}, \mathrm{~J}=7.3,1 \mathrm{H}$, ArH), 7.00-7.10 (m, 3H, ArH), $6.44\left(\mathrm{~s}, 1 \mathrm{H}, \mathbf{H}_{\text {aminal }}\right), 5.11\left(\mathrm{t}, \mathrm{J}=7.6 \mathrm{~Hz}, 1 \mathrm{H}, \mathrm{CH}-\mathrm{CH}_{2}\right), 4.28(\mathrm{dd}, \mathrm{J}=8.6,7.6 \mathrm{~Hz}$, $\left.1 \mathrm{H}, \mathrm{CH}_{2}\right), 3.71\left(\mathrm{dd}, \mathrm{J}=8.6,7.6 \mathrm{~Hz}, 1 \mathrm{H}, \mathrm{CH}_{2}\right), 2.32\left(\mathrm{~s}, 3 \mathrm{H}, \mathrm{CH}_{3}\right) ;{ }^{13} \mathrm{C} \mathrm{NMR}\left(500 \mathrm{MHz}, \mathrm{CDCl}_{3}\right) 138.2,137.5,136.7$, 134.8, 133.5, 130.4, 129.3, 129.0, 128.7, 128.2, 127.8, 127.2, 127.1, 126.6, 92.5, 73.1, 60.7, 19.6; HRMS (ESI $\left.{ }^{+}\right)$ calc'd for $\left[\mathrm{C}_{22} \mathrm{H}_{21} \mathrm{NO}_{3} \mathrm{SNa}\right]^{+}$requires $\mathrm{m} / z$ 402.1140, found $\mathrm{m} / \mathrm{z} 402.1141$. Isolated as a colorless oil. 


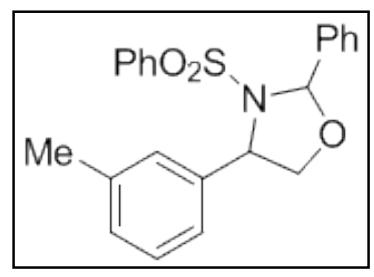

N-Benzensulfonyl-4-(3-methylphenyl)-2-phenyl-1,3-oxazolane (Table 2, entry 5). Prepared according to the general procedure using $142 \mathrm{mg} 2$-methylstyrene. Yield 1: $384 \mathrm{mg}$ (1.01 mmol, 84.5\% yield), 1.9:1 cis:trans; yield 2: $375 \mathrm{mg}$ (.987 mmol, 82.3\% yield), 1.9:1 cis:trans. Major diastereomer (2S*,4S*): IR (neat) 3063, 3032, 2921, 2884, 1351, 1165; ${ }^{1} \mathrm{H}$ NMR (500 MHz, $\left.\mathrm{CDCl}_{3}\right) 7.77$ (dd, J = 8.6, $1.0 \mathrm{~Hz}, 2 \mathrm{H}, \mathrm{SO}_{2} \mathrm{PhH}$ ), 7.56-7.62 (m, 3H, ArH), 7.48 (t, J = 7.7 Hz, 2H, ArH), 7.33-7.41 (m, 3H, ArH), $7.14(\mathrm{t}$, $\mathrm{J}=7.7,1 \mathrm{H}, \operatorname{ArH}), 7.03(\mathrm{~d}, \mathrm{~J}=7.7 \mathrm{~Hz}, 2 \mathrm{H}, \operatorname{ArH}), 6.99(\mathrm{~s}, 1 \mathrm{H}, \operatorname{ArH}), 6.37$ (s, 1H, $\left.\mathbf{H}_{\text {aminal }}\right), 4.84\left(\mathrm{t}, \mathrm{J}=7.1 \mathrm{~Hz}, 1 \mathrm{H}, \mathrm{CH}-\mathrm{CH}_{2}\right), 4.16\left(\mathrm{dd}, \mathrm{J}=8.8,7.4 \mathrm{~Hz}, 1 \mathrm{H}, \mathrm{CH}_{2}\right), 3.88(\mathrm{dd}, \mathrm{J}=8.8,6.6 \mathrm{~Hz}, 1 \mathrm{H}, \mathrm{CH})$, $2.24\left(\mathrm{~s}, 3 \mathrm{H}, \mathrm{CH}_{3}\right) ;{ }^{13} \mathrm{C} \mathrm{NMR}\left(500 \mathrm{MHz}, \mathrm{CDCl}_{3}\right)$ 138.5, 138.3, 138.2, 137.6, 133.4, 129.3, 129.0, 128.9, 128.7, 128.6, 128.2, 128.1, 127.4, 124.3, 92.7, 73.4, 63.4, 21.6; HRMS (ESI ${ }^{+}$) calc'd for $\left[\mathrm{C}_{22} \mathrm{H}_{21} \mathrm{NO}_{3} \mathrm{SNa}^{+}\right.$requires $m / z$ 402.1140, found $m / z$ 402.1145. Isolated as a white solid $\left(\mathrm{mp}=126-127^{\circ} \mathrm{C}\right)$. Minor diastereomer $\left(2 R^{*}, 4 S^{*}\right)$ : $\mathrm{IR}$ (neat) 3062, 3032, 2920, 2878, 1355, 1167; ${ }^{1} \mathrm{H}$ NMR (500 MHz, CDCl $)_{3} \delta 7.54$ (m, 2H, ArH), 7.31-7.43 (m, 4H, ArH), 7.10-7.21 (m, 4H, ArH), $7.06(\mathrm{~m}, 2 \mathrm{H}, \operatorname{ArH}), 7.01(\mathrm{~m}, 1 \mathrm{H}, \operatorname{ArH}), 6.94(\mathrm{~m}, 1 \mathrm{H}, \operatorname{ArH}), 6.37\left(\mathrm{~s}, 1 \mathrm{H}, \mathbf{H}_{\mathrm{aminal}}\right)$, $5.06\left(\mathrm{dd}, \mathrm{J}=6.4,1.9 \mathrm{~Hz}, 1 \mathrm{H}, \mathrm{CH}-\mathrm{CH}_{2}\right), 4.36\left(\mathrm{dd}, \mathrm{J}=8.8,6.4 \mathrm{~Hz}, 1 \mathrm{H}, \mathrm{CH}_{2}\right), 3.94(\mathrm{dd}, \mathrm{J}=8.8,1.9 \mathrm{~Hz}, 1 \mathrm{H}, \mathrm{CH})$, $2.15\left(\mathrm{~s}, 3 \mathrm{H}, \mathrm{CH}_{3}\right) ;{ }^{13} \mathrm{C} \mathrm{NMR}\left(500 \mathrm{MHz} \mathrm{CDCl}_{3}\right)$ 140.6, 138.7, 138.4(3), 138.4(1), 132.1, 129.3, 129.1, 128.5(7), 128.5(5), 128.5(0), 128.4, 127.5, 127.1, 125.5, 92.4, 73.1, 63.0, 21.4; HRMS (ESI ${ }^{+}$) calc'd for $\left[\mathrm{C}_{22} \mathrm{H}_{21} \mathrm{NO}_{3} \mathrm{SNa}^{+}\right.$ requires $\mathrm{m} / \mathrm{z} 402.1140$, found $\mathrm{m} / \mathrm{z} 402.1154$. Isolated as a colorless oil.

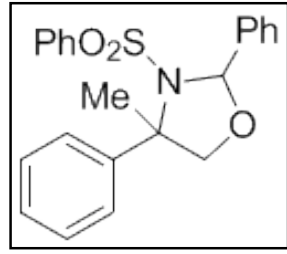

$\boldsymbol{N}$-Benzensulfonyl-4-methyl-2,4-diphenyl-1,3-oxazolane (Table 2, entry 6). To a dry 2 dram vial containing a stir bar was added $35 \mathrm{mg}(.12 \mathrm{mmol})$ copper(II) trifluoroacetate hydrate, followed by $627 \mathrm{mg}(2.4 \mathrm{mmol})$ trans-(2-phenylsulfonyl)-3-phenyloxaziridine (1) and $4.8 \mathrm{~mL}$ freshly distilled $\mathrm{CH}_{2} \mathrm{Cl}_{2}$. A second vial was charged with $142 \mathrm{mg}(1.2 \mathrm{mmol}) \alpha$ methylstyrene, $42 \mu \mathrm{L}$ HMPA $(240 \mu \mathrm{mol})$ and $4 \mathrm{~mL} \mathrm{CH}_{2} \mathrm{Cl}_{2}$. The styrene solution was then added via syringe to the oxaziridine solution, and the empty vial rinsed with two portions of $0.4 \mathrm{ml} \mathrm{CH}_{2} \mathrm{Cl}_{2}$. The vial was sealed with a septum, and the reaction was stirred under $\mathrm{N}_{2}$ for 2 $\mathrm{h}$ at $35{ }^{\circ} \mathrm{C}$. The solvent was then removed and the mixture was directly purified on silica gel using $6 \%$ to $9 \%$ EtOAc in hexanes as eluent to give the aminal product as a mixture of 2 diastereomers. Yield 1: $346 \mathrm{mg}(.910 \mathrm{mmol}$, $75.8 \%$ yield), $2.3: 1 \mathrm{dr}$; yield 2: $335 \mathrm{mg}$ (.883 mmol, $73.7 \%$ yield), 2.0:1 dr. Major diastereomer $\left(2 R^{*}, 4 S^{*}\right)$ : IR (neat) 3062, 3030, 2983, 2933, 2877, 1348, 1162; ${ }^{1} \mathrm{H}$ NMR $\left(500 \mathrm{MHz}, \mathrm{CDCl}_{3}\right) \delta 7.58(\mathrm{dd}, \mathrm{J}=7.8,2.0 \mathrm{~Hz}, 2 \mathrm{H},-$ $\left.\mathrm{SO}_{2} \mathrm{PhH}\right), 7.27-7.43(\mathrm{~m}, 6 \mathrm{H}, \mathrm{ArH}), 7.17(\mathrm{tt}, \mathrm{J}=7.3,1.1 \mathrm{~Hz}, 1 \mathrm{H}, \operatorname{ArH}), 7.06-7.13(\mathrm{~m}, 6 \mathrm{H}, \operatorname{ArH}), 6.46(\mathrm{~s}, 1 \mathrm{H}$, $\left.\mathbf{H}_{\text {aminal }}\right), 4.08\left(\mathrm{~d}, \mathrm{~J}=9.0 \mathrm{~Hz}, 1 \mathrm{H}, \mathrm{CH}_{2}\right), 3.94\left(\mathrm{~d}, \mathrm{~J}=9.0 \mathrm{~Hz}, 1 \mathrm{H}, \mathrm{CH}_{2}\right), 2.05(\mathrm{~s}, 3 \mathrm{H}, \mathrm{CH}) ;{ }^{13} \mathrm{C} \mathrm{NMR}(500 \mathrm{MHz}$, $\left.\mathrm{CDCl}_{3}\right)$ 141.4, 140.7, 138.7, 131.9, 129.0, 128.5, 128.4(9), 128.4, 127.9, 127.8, 127.2, 93.2, 80.8, 67.0, 24.5; HRMS $\left(\mathrm{ESI}^{+}\right)$calc'd for $\left[\mathrm{C}_{22} \mathrm{H}_{21} \mathrm{NO}_{3} \mathrm{SNa}\right]^{+}$requires $\mathrm{m} / \mathrm{z} 402.1140$, found $\mathrm{m} / \mathrm{z} 402.1143$. Isolated as a white solid $(\mathrm{mp}=$ $127-128^{\circ} \mathrm{C}$ ). Minor diastereomer $\left(2 S^{*}, 4 S^{*}\right)$ : IR (neat) 3062, 3032, 2925, 2881, 1345, $1160 ;{ }^{1} \mathrm{H} \mathrm{NMR}(500 \mathrm{MHz}$, $\left.\mathrm{CDCl}_{3}\right) \delta 7.63\left(\mathrm{~m}, 2 \mathrm{H},-\mathrm{SO}_{2} \mathrm{PhH}\right), 7.20-7.43(\mathrm{~m}, 9 \mathrm{H}, \mathrm{ArH}), 7.07-7.17$ (m, 4H, ArH), 6.27 (s, 1H, $\left.\mathbf{H}_{\text {aminal }}\right), 4.38(\mathrm{~d}, \mathrm{~J}$ $\left.=8.8 \mathrm{~Hz}, 1 \mathrm{H}, \mathrm{CH}_{2}\right), 4.00\left(\mathrm{~d}, \mathrm{~J}=8.8 \mathrm{~Hz}, 1 \mathrm{H}, \mathrm{CH}_{2}\right), 2.15\left(\mathrm{~s}, 3 \mathrm{H}, \mathrm{CH}_{3}\right) ;{ }^{13} \mathrm{C} \mathrm{NMR}\left(500 \mathrm{MHz}, \mathrm{CDCl}_{3}\right) 142.0,141.3$, 137.3, 132.3, 129.4, 128.5(6), 128.5(5), 128.4, 128.3, 127.9, 127.6, 127.0, 93.0, 81.3, 69.0, 24.2; HRMS (EI $\left.{ }^{+}\right)$calc'd for $\left[\mathrm{C}_{22} \mathrm{H}_{21} \mathrm{NO}_{3} \mathrm{SNa}\right]^{+}$requires $\mathrm{m} / z$ 402.1140, found $\mathrm{m} / z$ 402.1151. Isolated as a colorless oil.

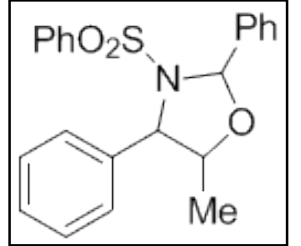

N-Benzensulfonyl-5-methyl-2,4-diphenyl-1,3-oxazolane (Table 2, entry 7). Prepared according to the general procedure using $142 \mathrm{mg} \beta$-methylstyrene. Yield 1: $397 \mathrm{mg}$ (1.05 mmol, 87.1\% yield), 1.2:1 dr; yield 2: $380 \mathrm{mg}$ (1.00 mmol, 83.3\% yield), 1.0:1 dr. Major diastereomer $\left(2 S^{*}, 4 S^{*}, 5 S^{*}\right)$ : IR (neat) $3063,3031,2977,2931,1355,1168 ;{ }^{1} \mathrm{H}$ NMR $(500$ $\left.\mathrm{MHz} \mathrm{CDCl}_{3}\right) \delta 7.83\left(\mathrm{~d}, \mathrm{~J}=8.1 \mathrm{~Hz}, 2 \mathrm{H},-\mathrm{SO}_{2} \mathrm{PhH}\right), 7.70(\mathrm{~d}, \mathrm{~J}=8.1 \mathrm{~Hz}, 2 \mathrm{H}, \mathrm{ArH}), 7.61(\mathrm{t}, \mathrm{J}=$ $7.4 \mathrm{~Hz}, 1 \mathrm{H}, \operatorname{ArH}), 7.51(\mathrm{t}, \mathrm{J}=7.8 \mathrm{~Hz}, 2 \mathrm{H}, \operatorname{ArH}), 7.43(\mathrm{t}, \mathrm{J}=7.4 \mathrm{~Hz}, 2 \mathrm{H}, \operatorname{ArH}), 7.37(\mathrm{t}, \mathrm{J}=7.4$, $1 \mathrm{H}, \operatorname{ArH}), 7.19-7.25(\mathrm{~m}, 3 \mathrm{H}, \operatorname{ArH}), 7.10-7.16(\mathrm{~m}, 2 \mathrm{H}, \operatorname{ArH}), 6.50\left(\mathrm{~s}, 1 \mathrm{H}, \mathbf{H}_{\text {aminal }}\right), 4.13(\mathrm{~d}, \mathrm{~J}=$ $8.1 \mathrm{~Hz}, 1 \mathrm{H}, \mathrm{Ph}-\mathrm{CH}-\mathrm{CH}), 3.99$ (dq, J = 8.2, $6.1 \mathrm{~Hz}, 1 \mathrm{H}, \mathrm{CH}-\mathrm{CH} 3), 1.14$ (d, J = 6.1 Hz, 3H, CH $)$; ${ }^{13} \mathrm{C} \mathrm{NMR} \mathrm{(500}$ $\left.\mathrm{MHz}_{,} \mathrm{CDCl}_{3}\right)$ 139.2, 137.5, 137.0, 133.5, 129.3, 128.7(2), 128.7(2) 128.3, 128.2, 127.5, 126.9, 91.4, 81.3, 71.1, 17.1; HRMS $\left(\mathrm{ESI}^{+}\right.$) calc'd for $\left[\mathrm{C}_{22} \mathrm{H}_{21} \mathrm{NO}_{3} \mathrm{SH}\right]^{+}$requires $\mathrm{m} / z$ 378.1164, found $\mathrm{m} / \mathrm{z} 378.1178$. Isolated as a colorless oil. Minor diastereomer $\left(2 R^{*}, 4 S^{*}, 5 S^{*}\right)$ : IR (neat) 3065, 3037, 2981, 2877, 1348, 1164; ${ }^{1} \mathrm{H} \mathrm{NMR}(500 \mathrm{MHz}$, $\left.\mathrm{CDCl}_{3}\right) \delta 7.43(\mathrm{~d}, \mathrm{~J}=7.4 \mathrm{~Hz}, 2 \mathrm{H}, \mathrm{ArH}), 7.30-7.39(\mathrm{~m}, 7 \mathrm{H}, \mathrm{ArH}), 7.23(\mathrm{t}, \mathrm{J}=7.9 \mathrm{~Hz}, 2 \mathrm{H}, \operatorname{ArH}), 7.14(\mathrm{t}, \mathrm{J}=7.5 \mathrm{~Hz}$, 2H, ArH), 7.01 (d, J = 8.7, 2H, ArH), 6.26 (s, 1H, Haminal), 4.44 (d, J = 7.7 Hz, 1H, CH-CH-CH $)_{3}, 4.26(\mathrm{dq}, \mathrm{J}=7.6$, $\left.6.1 \mathrm{~Hz}, 1 \mathrm{H}, \mathrm{CH}-\mathrm{CH}-\mathrm{CH}_{3}\right), 1.37\left(\mathrm{~d}, \mathrm{~J}=6.1 \mathrm{~Hz}, 3 \mathrm{H}, \mathrm{CH}_{3}\right) ;{ }^{13} \mathrm{C} \mathrm{NMR}\left(500 \mathrm{MHz}, \mathrm{CDCl}_{3}\right) 142.1,141.4,138.2,135.5$, 132.2, 129.5, 129.3, 128.5, 128.4, 128.3, 128.2, 127.8, 127.2, 125.2, 91.5, 81.4, 68.6, 38.15; HRMS (ESI ${ }^{+}$) calc'd for $\left[\mathrm{C}_{22} \mathrm{H}_{21} \mathrm{NO}_{3} \mathrm{SNa}\right]^{+}$requires $\mathrm{m} / \mathrm{z} 402.1140$, found $\mathrm{m} / \mathrm{z} 402.1130$. Isolated as a colorless oil. 


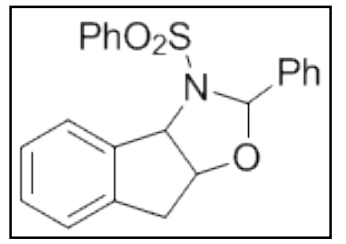

N-Benzenesulfonyl-2-phenyl-2,3,3a,8-tetrahydro-indeno[1,2-d]oxazole (Table 2, entry 8). Prepared according to the general procedure using $141 \mathrm{mg}$ indene and purified on silica gel with 2:1 $\mathrm{CH}_{2} \mathrm{Cl}_{2}$ :hexanes as eluent. Yield 1: $358 \mathrm{mg}$ (.948 mmol, $79.3 \%$ yield), 3.8:1 dr; yield 2: $379 \mathrm{mg}$ (1.00 mmol, 83.0\% yield), 2.7:1 dr. Major diastereomer $\left(2 S^{*}, 3 \mathrm{a} S^{*}, 8 \mathrm{a} R^{*}\right)$ : IR (neat) $3066,3037,2925,1346,1161 ;{ }^{1} \mathrm{H}$ NMR $\left(500 \mathrm{MHz}, \mathrm{CDCl}_{3}\right) \delta$ $7.91\left(\mathrm{dd}, \mathrm{J}=8.2,1.1 \mathrm{~Hz}, 2 \mathrm{H},-\mathrm{SO}_{2} \mathrm{PhH}\right), 7.63(\mathrm{tt}, \mathrm{J}=7.4,1.1 \mathrm{~Hz}, 1 \mathrm{H}, \mathrm{ArH}), 7.50-7.59(\mathrm{~m}$, $3 \mathrm{H}, \operatorname{ArH}), 7.46(\mathrm{dd}, \mathrm{J}=5.4,3.9 \mathrm{~Hz}, 1 \mathrm{H}, \mathrm{ArH}), 7.17-7.26(\mathrm{~m}, 2 \mathrm{H}, \mathrm{ArH}), 7.02-7.16(\mathrm{~m}, 6 \mathrm{H}$, $\mathrm{ArH}), 6.12\left(\mathrm{~s}, 1 \mathrm{H}, \mathbf{H}_{\text {aminal }}\right), 5.46\left(\mathrm{~d}, \mathrm{~J}=5.6 \mathrm{~Hz}, 1 \mathrm{H}, \mathrm{CH}-\mathrm{CH}-\mathrm{CH}_{2}\right), 4.48\left(\mathrm{t}, \mathrm{J}=5.1 \mathrm{~Hz}, 1 \mathrm{H}, \mathrm{CH}-\mathrm{CH}-\mathrm{CH}_{2}\right), 3.09(\mathrm{~d}, \mathrm{~J}=$ $\left.17.4,1 \mathrm{H}, \mathrm{CH}_{2}\right), 3.02\left(\mathrm{dd}, 17.4,5.1 \mathrm{~Hz}, 1 \mathrm{H}, \mathrm{CH}_{2}\right) ;{ }^{13} \mathrm{C}$ NMR $\left(500 \mathrm{MHz}, \mathrm{CDCl}_{3}\right) 142.7,142.4,140.9,140.8,136.1$, 132.0, 131.5, 131.4, 130.6(3), 130.6(1), 130.2, 129.7, 128.8, 127.8, 96.0, 84.7, 70.5, 40.1; HRMS (ESI $\left.{ }^{+}\right)$calc'd for $\left[\mathrm{C}_{22} \mathrm{H}_{19} \mathrm{NO}_{3} \mathrm{SNa}\right]^{+}$requires $\mathrm{m} / \mathrm{z} 400.0983$, found $\mathrm{m} / \mathrm{z} 400.0993$. Isolated as a white solid $\left(\mathrm{mp}=149.5-150.3{ }^{\circ} \mathrm{C}\right)$. Minor diastereomer $\left(2 R^{*}, 3 \mathrm{a} S^{*}, 8 \mathrm{a} R^{*}\right)$ : IR (neat) $3066,3036,2923,2831,1353,1168 ;{ }^{1} \mathrm{H} \mathrm{NMR}\left(500 \mathrm{MHz}, \mathrm{CDCl}_{3}\right) \delta$ $8.02(\mathrm{~m}, 1 \mathrm{H}, \operatorname{ArH}) 7.31-7.41$ (m, 3H, ArH), 7.24-7.31 (m, 2H, ArH), 7.15-7.24 (m, 6H, ArH), 7.08-7.15 (t, J = 7.8 $\mathrm{Hz}, 2 \mathrm{H}, \mathrm{ArH}), 5.83\left(\mathrm{~s}, 1 \mathrm{H}, \mathbf{H}_{\text {aminal }}\right), 5.74\left(\mathrm{~d}, \mathrm{~J}=5.6 \mathrm{~Hz}, 1 \mathrm{H}, \mathrm{CH}-\mathrm{CH}-\mathrm{CH}_{2}\right), 5.13(\mathrm{td}, \mathrm{J}=5.6,1.7 \mathrm{~Hz}, 1 \mathrm{H}, \mathrm{CH}-\mathrm{CH}-$ $\left.\mathrm{CH}_{2}\right), 3.18\left(\mathrm{dd}, \mathrm{J}=17.5,5.8 \mathrm{~Hz}, 1 \mathrm{H}, \mathrm{CH}_{2}\right), 3.07\left(\mathrm{~d}, \mathrm{~J}=17.5,1 \mathrm{H}, \mathrm{CH}_{2}\right) ;{ }^{13} \mathrm{C}$ NMR $\left(500 \mathrm{MHz}, \mathrm{CDCl}_{3}\right) 142.1,141.4$, $138.2,135.5,132.2,129.5,129.3,128.5,128.4,128.3,128.2,127.8,127.2,125.2,91.5,81.4,68.6,38.2$; HRMS $\left(\mathrm{ESI}^{+}\right)$calc'd for $\left[\mathrm{C}_{22} \mathrm{H}_{19} \mathrm{NO}_{3} \mathrm{SNa}\right]^{+}$requires $\mathrm{m} / \mathrm{z} 400.0983$, found $\mathrm{m} / \mathrm{z} 400.0992$. Isolated as a white solid $(\mathrm{mp}=$ $\left.173.5-174{ }^{\circ} \mathrm{C}\right)$.

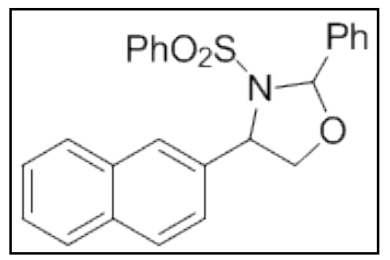

$N$-Benzensulfonyl-4-(2-naphthyl)-2-phenyl-1,3-oxazolane (Table 2 , entry 9). Prepared according to the general procedure using $186 \mathrm{mg}$ 2-vinylstyrene and purified on silica gel with 1:6 EtOAc:hexanes as eluent. The imine resulting from reduction of Davis's oxaziridine was inseparable from the product by flash chromatography. The clean product was obtained by treatment of the purified product and imine with $4.5 \mathrm{mg}$

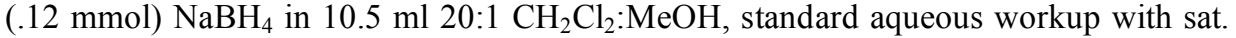
$\mathrm{NaHCO}_{3}$, and chromatographic purification using 1:6 EtOAc:hexanes as eluent. Yield 1: $421 \mathrm{mg}$ (1.01 mmol, 83.8\% yield), 1.6:1 cis:trans; yield 2: $413 \mathrm{mg}$ (.994 mmol, 82.5\% yield), 1.6:1 cis:trans. Major diastereomer $\left(2 S^{*}, 4 S^{*}\right)$ : IR (neat) $3060,2934,2878,1354,1167 ;{ }^{1} \mathrm{H}$ NMR $\left(500 \mathrm{MHz}, \mathrm{CDCl}_{3}\right) \delta 7.67-7.81$ $(\mathrm{m}, 6 \mathrm{H}, \operatorname{Ar} \mathbf{H}), 7.63(\mathrm{~d}, \mathrm{~J}=7.1 \mathrm{~Hz}, 2 \mathrm{H}, \operatorname{ArH}), 7.55(\mathrm{t}, \mathrm{J}=7.7,1 \mathrm{H}, \operatorname{Ar} \mathbf{H}), 7.41-7.48(\mathrm{~m}, 4 \mathrm{H}, \operatorname{ArH}), 7.29-7.41(\mathrm{~m}$, 4H, $\operatorname{ArH}), 6.41\left(\mathrm{~s}, 1 \mathrm{H}, \mathbf{H}_{\text {aminal }}\right), 5.07\left(\mathrm{t}, \mathrm{J}=7.0 \mathrm{~Hz}, 1 \mathrm{H}, \mathrm{CH}_{-} \mathrm{CH}_{2}\right), 4.23\left(\mathrm{dd}, \mathrm{J}=9.2,7.1 \mathrm{~Hz}, 1 \mathrm{H}, \mathrm{C} \mathbf{H}_{2}\right), 4.00(\mathrm{~d}, \mathrm{~J}=$ 9.1, 6.5, $\left.1 \mathrm{H}, \mathrm{CH}_{2}\right) ;{ }^{13} \mathrm{C}$ NMR $\left(500 \mathrm{MHz}, \mathrm{CDCl}_{3}\right)$ 138.0, 137.7, 136.0, 133.4, 133.2, 133.1(5), 129.3, 129.1, 128.8 , $128.7,128.2,128.1,127.8,127.4,126.8,126.5,126.4,124.7,92.8,73.3,63.5$; HRMS $\left(\mathrm{ESI}^{+}\right)$calc'd for $\left[\mathrm{C}_{25} \mathrm{H}_{21} \mathrm{NO}_{3} \mathrm{SNa}\right]^{+}$requires $\mathrm{m} / \mathrm{z} 438.1140$, found $\mathrm{m} / \mathrm{z} 438.1161$. Isolated as a white solid $\left(\mathrm{mp}=195-198{ }^{\circ} \mathrm{C}\right)$. Minor diastereomer $\left(2 R^{*}, 4 S^{*}\right)$ : IR (neat) 3060, 2918, 1349, 1164; ${ }^{1} \mathrm{H}$ NMR $\left(500 \mathrm{MHz}, \mathrm{CDCl}_{3}\right) \delta$ 7.70-7.79 (m, 3H, $\mathrm{ArH}$ ), $7.58(\mathrm{dd}, \mathrm{J}=7.8,2.1 \mathrm{~Hz}, 2 \mathrm{H}, \mathrm{ArH}), 7.54(\mathrm{~d}, \mathrm{~J}=8.5 \mathrm{~Hz}, 1 \mathrm{H}, \mathrm{ArH}), 7.48(\mathrm{~m}, 2 \mathrm{H}, \operatorname{ArH}), 7.39-7.44(\mathrm{~m}, 3 \mathrm{H}, \operatorname{ArH}), 7.28$ $(\mathrm{dd}, \mathrm{J}=8.8,2.1 \mathrm{~Hz}, 1 \mathrm{H}, \operatorname{ArH}), 7.16(\mathrm{tt}, \mathrm{J}=7.3,1.1 \mathrm{~Hz}, 1 \mathrm{H}, \operatorname{ArH}), 7.10(\mathrm{dd}, \mathrm{J}=8.4,1.1 \mathrm{~Hz}, 2 \mathrm{H}, \operatorname{ArH}), 6.86(\mathrm{t}, \mathrm{J}=$ $8.0 \mathrm{~Hz}, 2 \mathrm{H}, \mathrm{ArH}), 6.47\left(\mathrm{~s}, 1 \mathrm{H}, \mathbf{H}_{\text {aminal }}\right), 5.25$ (dd, J = 6.5, 2.0 Hz, 1H, CH-CH $)_{2}, 4.45\left(\mathrm{dd}, \mathrm{J}=9.2,6.5 \mathrm{~Hz}, 1 \mathrm{H}, \mathrm{CH}_{2}\right)$, 4.07 (dd, J = 9.2, $\left.2.0 \mathrm{~Hz}, 1 \mathrm{H}, \mathrm{CH}_{2}\right) ;{ }^{13} \mathrm{C}$ NMR $\left(500 \mathrm{MHz}, \mathrm{CDCl}_{3}\right)$ 140.5, 138.6, 135.8, 133.2, 133.1, 132.0, 129.4, 128.7, 128.6, 128.2(7), 128.2(5), 127.8, 127.7, 127.5, 127.0, 126.5, 126.4(8), 125.1, 92.6, 73.0, 63.1; $\mathrm{HRMS}_{\left(\mathrm{ESI}^{+}\right)}$ calc'd for $\left[\mathrm{C}_{25} \mathrm{H}_{21} \mathrm{NO}_{3} \mathrm{SNa}\right]^{+}$requires $m / z 438.1140$, found $m / z$ 438.1119. Isolated as a colorless oil.

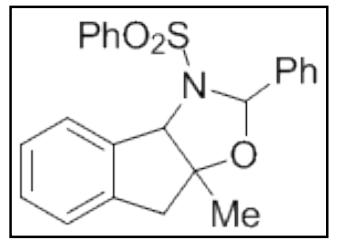

$\mathrm{N}$-Benzensulfonyl-8a-methyl-2-phenyl-2,3,3a,8-tetrahydro-indeno[1,2-d]oxazole (Table 2, entry 10). To a dry $25 \mathrm{~mL}$ round bottom flask containing a stir bar was added 35 $\mathrm{mg}(.12 \mathrm{mmol})$ copper(II) trifluoroacetate hydrate, followed by $627 \mathrm{mg}(2.4 \mathrm{mmol})$ trans(2-phenylsulfonyl)-3-phenyloxaziridine (1) and $4.8 \mathrm{~mL}$ freshly distilled $\mathrm{CH}_{2} \mathrm{Cl}_{2}$. A second vial was charged with $157 \mathrm{mg}(1.2 \mathrm{mmol}) 2$-methylindene, $42 \mu \mathrm{L}$ HMPA $(240 \mu \mathrm{mol})$ and 4 $\mathrm{mL} \mathrm{CH}_{2} \mathrm{Cl}_{2}$. The indene solution was then added via syringe to the oxaziridine solution,

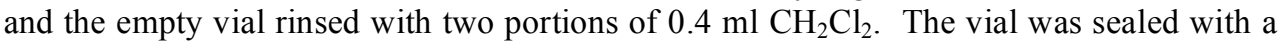
septum, and the reaction was stirred under $\mathrm{N}_{2}$ for $3.5 \mathrm{~h}$ at $35^{\circ} \mathrm{C}$. Dimethylsulfide $(.1 \mathrm{ml}, 1.36 \mathrm{mmol})$ was then added to the reaction to reduce any remaining oxaziridine. The solvent was then removed and the mixture was directly purified on silica gel using 10\% EtOAc and 2\% triethylamine in hexanes with as eluent to give the aminal product as a mixture of two diastereomers. Yield 1: $398 \mathrm{mg}(1.02 \mathrm{mmol}, 84.0 \%$ yield), 1.6:1 dr; yield 2: $396 \mathrm{mg}$ $\left(1.01 \mathrm{mmol}, 83.7 \%\right.$ yield), 1.6:1 dr. Major diastereomer $\left(2 \mathrm{R}^{*}, 3 \mathrm{aR}^{*}, 8 \mathrm{aS}\right)$ : IR (neat) 3068, 3035, 2970, 2929, 2875, 1354, 1168; ${ }^{1} \mathrm{H}$ NMR (500 MHz, $\left.\mathrm{CDCl}_{3}\right) \delta$ 7.90-7.94 (m, 2H, ArH), 7.63 (tt, J = 7.5, $\left.1.2 \mathrm{~Hz}, 1 \mathrm{H}, \operatorname{ArH}\right)$, 7.52-7.58 (m, 2H, ArH), 7.46-7.51 (m, 1H, ArH), 7.24-7.30 (m, 2H, ArH), $7.18(\mathrm{tt}, \mathrm{J}=7.2,1.4 \mathrm{~Hz}, 1 \mathrm{H}, \operatorname{ArH})$, 7.09-7.15 (m, 3H, ArH), 7.00-7.04 (m, 2H, ArH), 6.07 (s, 1H, Haminal), 5.31 (s, 1H, N-CH-C), 3.17 (d, J = 17.1 $\left.\mathrm{Hz}, 1 \mathrm{H}, \mathrm{CH}_{2}\right), 2.90\left(\mathrm{~d}, \mathrm{~J}=17.1 \mathrm{~Hz}, 1 \mathrm{H}, \mathrm{CH}_{2}\right), 1.05\left(\mathrm{~s}, 3 \mathrm{H}, \mathrm{CH}_{3}\right) ;{ }^{13} \mathrm{C} \mathrm{NMR}\left(500 \mathrm{MHz}, \mathrm{CDCl}_{3}\right)$ 141.0, 140.5, 138.6, 
138.5(5), 133.5, 129.5, 129.1, 129.0, 128.3, 128.2, 127.8, 127.6, 126.5 125.3, 92.5, 90.9, 72.0, 43.8, 21.7; HRMS $\left(\mathrm{ESI}^{+}\right)$calc'd for $\left[\mathrm{C}_{23} \mathrm{H}_{21} \mathrm{NO}_{3} \mathrm{SNa}\right]^{+}$requires $\mathrm{m} / z$ 414.1140, found $\mathrm{m} / \mathrm{z}$ 414.1147. Isolated as a white solid $(\mathrm{mp}=$ $\left.154-155^{\circ} \mathrm{C}\right)$. Minor diastereomer $\left(2 \mathrm{~S}^{*}, 3 \mathrm{aR}^{*}, 8 \mathrm{aS} *\right)$ : IR (neat) $3068,3039,2971,2928,1338,1160 ;{ }^{1} \mathrm{H}$ NMR $(500$ $\left.\mathrm{MHz}, \mathrm{CDCl}_{3}\right) \delta$ 7.97-7.90 (m, 2H, ArH), 7.32-7.40 (m, 3H, ArH), 7.22-7.25 (m, 2H, ArH), 7.13-7.20 (m, 6H, ArH), 7.04-7.09 (m, 2H, ArH), $5.62\left(\mathrm{~s}, 1 \mathrm{H}, \mathbf{H}_{\text {aminal }}\right), 5.42\left(\mathrm{~s}, 1 \mathrm{H}, \mathrm{O}-\mathrm{CH}-\mathrm{CH}_{3}\right), 3.25(\mathrm{~d}, \mathrm{~J}=17.1 \mathrm{~Hz}, 1 \mathrm{H}, \mathrm{CH} 2), 3.05$ $\left(\mathrm{d}, \mathrm{J}=17.1 \mathrm{~Hz}, 1 \mathrm{H}, \mathrm{CH}_{2}\right), 1.67$ (s, 3H, $\left.\mathrm{CH}_{3}\right) ;{ }^{13} \mathrm{C}$ NMR (500 MHz, $\left.\mathrm{CDCl}_{3}\right)$ 142.5, 141.5, 138.4, 134.4, 132.4, 129.6, $129.5,129.1,128.4,128.3,128.0,127.9,127.2,125.0,91.0,89.3,73.7,44.4,25.6$; HRMS $\left(\mathrm{EI}^{+}\right)$calc'd for $\left[\mathrm{C}_{23} \mathrm{H}_{21} \mathrm{NO}_{3} \mathrm{SNa}\right]^{+}$requires $\mathrm{m} / z 414.1140$, found $\mathrm{m} / \mathrm{z} 414.1160$. Isolated as a clear solid $\left(\mathrm{mp}=156-158{ }^{\circ} \mathrm{C}\right)$.

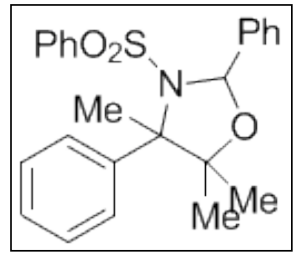

$\boldsymbol{N}$-Benzensulfonyl-4,5,5-trimethyl-2,4-diphenyl-1,3-oxazolane (Table 2, entry 11). To a dry $25 \mathrm{~mL}$ round bottom flask containing a stir bar was added $35 \mathrm{mg}(.12 \mathrm{mmol})$ copper(II) trifluoroacetate hydrate, followed by $627 \mathrm{mg}(2.4 \mathrm{mmol})$ trans-(2-phenylsulfonyl)-3phenyloxaziridine (1) and $4.8 \mathrm{~mL}$ freshly distilled $\mathrm{CH}_{2} \mathrm{Cl}_{2}$. A second vial was charged with $177 \mathrm{mg}(1.2 \mathrm{mmol}) \alpha, \beta, \beta$-trimethylstyrene, ${ }^{2} 42 \mu \mathrm{L}$ HMPA $(240 \mu \mathrm{mol})$ and $4 \mathrm{~mL} \mathrm{CH}_{2} \mathrm{Cl}_{2}$. The styrene solution was then added via syringe to the oxaziridine solution, and the empty vial

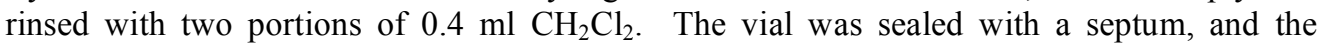
reaction was stirred under $\mathrm{N}_{2}$ for $2 \mathrm{~h}$ at $35^{\circ} \mathrm{C}$. The solvent was then removed and the mixture was directly purified on silica gel using $10 \%$ EtOAc and $2 \%$ triethylamine in hexanes with as eluent to give the aminal product as a mixture of two diastereomers. Yield 1: $395 \mathrm{mg}$ (0.97 mmol, $80.5 \%$ yield), 12.5:1 dr; yield 2: $400 \mathrm{mg}$ (.98 mmol, $80.6 \%$ yield), 10.2:1 dr. Major diastereomer $\left(2 \mathrm{~S}^{*}, 4 \mathrm{~S}^{*}\right)$ : IR (neat) 3092, 3062, 2999, 2937, 1347, 1161; ${ }^{1} \mathrm{H}$ NMR $\left(500 \mathrm{MHz}, \mathrm{CDCl}_{3}\right) \delta$ 7.50-7.56 (m, 4H, ArH), 7.21-7.37 (m, 9H, ArH), 7.13-7.17 (m, 2H, ArH), $6.18(\mathrm{~s}, 1 \mathrm{H}$, $\left.\mathbf{H}_{\text {aminal }}\right), 2.07\left(\mathrm{~s}, 3 \mathrm{H}, \mathrm{N}-\mathrm{C}-\mathrm{CH}_{3}\right), 1.37\left(\mathrm{~s}, 3 \mathrm{H}, \mathrm{O}-\mathrm{C}-\mathrm{CH}_{3}\right), .87\left(\mathrm{~s}, 3 \mathrm{H}, \mathrm{O}-\mathrm{C}-\mathrm{CH}_{3}\right) ;{ }^{13} \mathrm{C} \mathrm{NMR}\left(500 \mathrm{MHz}, \mathrm{CDCl}_{3}\right) 142.5$, 141.2, 136.8, 132.3, 129.6, 129.5, 128.3, 128.2, 127.9, 127.7, 127.6(7), 127.4, 90.3, 86.2, 74.7, 26.8, 23.7, 23.0; HRMS (ESI ${ }^{+}$) calc'd for $\left[\mathrm{C}_{24} \mathrm{H}_{25} \mathrm{NO}_{3} \mathrm{~S}\right]^{+}$requires $\mathrm{m} / \mathrm{z} 439.1453$, found $\mathrm{m} / z$ 439.1445. Isolated as a white solid ( $\mathrm{mp}=$ $\left.128-132{ }^{\circ} \mathrm{C}\right)$.

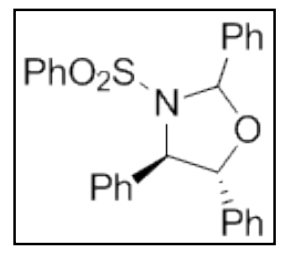

$\boldsymbol{N}$-Benzensulfonyl-2,4,5-triphenyl-1,3-oxazolane (Scheme 1). From trans-stilbene: To a dry $25 \mathrm{~mL}$ round bottom flask containing a stir bar was added $80.3 \mathrm{mg}(.277 \mathrm{mmol})$ copper(II) trifluoroacetate hydrate, $500 \mathrm{mg}(2.77 \mathrm{mmol})$ trans-stilbene, and $1.087 \mathrm{~g}(4.16 \mathrm{mmol})$ trans- $(2-$ phenylsulfonyl)-3-phenyloxaziridine (1). A dry two-dram vial was charged with $103.5 \mu \mathrm{L}$

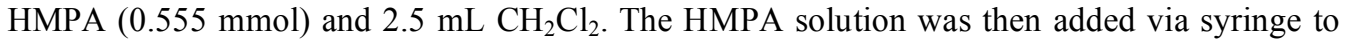

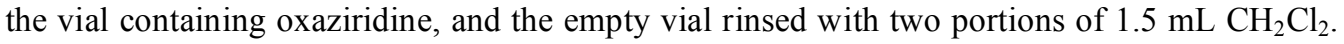
The vial was sealed with a septum, and the reaction was stirred under $\mathrm{N}_{2}$ for 12 hours. The reaction mixture was then diluted with $2 \mathrm{~mL} \mathrm{CH}_{2} \mathrm{Cl}_{2}$ and $5 \mathrm{~mL}$ saturated $\mathrm{NaHCO}_{3}$ solution. The layers were separated and the aqueous layer was washed twice with $\mathrm{CH}_{2} \mathrm{Cl}_{2}$. The combined organic layers were dried with $\mathrm{Na}_{2} \mathrm{SO}_{4}$ before being purifed on silica gel using 1:9 EtOAc:hexanes as eluent. Yield: $0.912 \mathrm{~g}(2.08 \mathrm{mmol}, 75 \%)$, 1.93:1 dr. From cis-stilbene: To a dry 2 dram vial containing a stir bar was added $80.3 \mathrm{mg}$ (.277 mmol) copper(II) trifluoroacetate hydrate, followed by $1.087 \mathrm{~g}(4.16 \mathrm{mmol})$ trans-(2-phenylsulfonyl)-3-phenyloxaziridine (1). A second vial was charged with $500 \mathrm{mg}$ cis-stilbene $(2.77 \mathrm{mmol}), 103.5 \mu \mathrm{L}$ HMPA $(0.555 \mathrm{mmol})$ and $2.5 \mathrm{~mL} \mathrm{CH}_{2} \mathrm{Cl}_{2}$. The stilbene solution was then added via syringe to the vial containing oxaziridine, and the empty vial rinsed with two portions of $1.5 \mathrm{~mL} \mathrm{CH}_{2} \mathrm{Cl}_{2}$. The vial was sealed with a septum, and the reaction was stirred under $\mathrm{N}_{2}$ for 24 hours. The reaction mixture was then diluted with $2 \mathrm{~mL} \mathrm{CH}_{2} \mathrm{Cl}_{2}$ and $5 \mathrm{~mL}$ saturated $\mathrm{NaHCO}_{3}$ solution. The layers were separated and the aqueous layer washed twice with $\mathrm{CH}_{2} \mathrm{Cl}_{2}$. The combined organic layers were then dried with $\mathrm{Na}_{2} \mathrm{SO}_{4}$ before being purifed on silica gel using 1:9 EtOAc:hexanes as eluent. Yield: $705.3 \mathrm{mg}$ (1.60 mmol, 58\%), $1.95: 1 \mathrm{dr}$. Major diastereomer $\left(2 S^{*}, 4 R^{*}, 5 R^{*}\right)$ : IR (neat) $3055,2987,1353,1266,1166 ;{ }^{1} \mathrm{H}$ NMR $(500 \mathrm{MHz}$, $\left.\mathrm{CDCl}_{3}\right) \delta 7.43(\mathrm{~d}, \mathrm{~J}=7.9 \mathrm{~Hz}, 2 \mathrm{H}, \operatorname{ArH}), 7.34-7.38(\mathrm{~m}, 7 \mathrm{H}, \operatorname{ArH}), 7.24-7.31(\mathrm{~m}, 5 \mathrm{H}, \operatorname{ArH}), 7.13-7.17(\mathrm{~m}, 4 \mathrm{H}$, ArH), 7.01 (d, J = 8.3 Hz, 2H, ArH), 6.40 (s, 1h, $\left.\mathbf{H}_{\text {aminal }}\right), 5.15$ (d, J = 8.3 Hz, 1H, N-CH-CH-O), 4.74 (d, J = 8.3 Hz, $1 \mathrm{H}, \mathrm{O}-\mathrm{CH}-\mathrm{CH}-\mathrm{N}) ;{ }^{13} \mathrm{C}$ NMR (500 MHz, CDCl3) 140.4, 136.8, 136.4, 135.6, 131.8, 129.6, 129.2, 128.7, 128.5 , 128.5, 128.4, 128.3, 128.2, 128.0, 126.8, 126.3, 93.4, 87.6, 70.6; HRMS (ESI ${ }^{+}$) calc'd for $\left[\mathrm{C}_{27} \mathrm{H}_{23} \mathrm{NO}_{3} \mathrm{SNa}^{+}\right.$requires $\mathrm{m} / \mathrm{z}$ 464.1296, found $\mathrm{m} / \mathrm{z}$ 464.1283. Isolated as a white solid $\left(\mathrm{mp}=167-169{ }^{\circ} \mathrm{C}\right)$. Minor diastereomer $\left(2 R^{*}, 4 R^{*}, 5 R^{*}\right)$; IR (neat) $3063,3032,3922,2852,1357,1169 ;{ }^{1} \mathrm{H}$ NMR $\left(500 \mathrm{MHz}, \mathrm{CDCl}_{3}\right) \delta 7.93(\mathrm{~d}, \mathrm{~J}=6.5 \mathrm{~Hz}$, 2H, ArH), $7.77(\mathrm{~d}, \mathrm{~J}=8.3 \mathrm{~Hz}, 2 \mathrm{H}, \operatorname{ArH}), 7.72(\mathrm{t}, \mathrm{J}=7.4 \mathrm{~Hz}, 1 \mathrm{H}, \operatorname{ArH}), 7.60(\mathrm{t}, \mathrm{J}=8.3 \mathrm{~Hz}, 2 \mathrm{H}, \operatorname{ArH}), 7.43(\mathrm{t}, \mathrm{J}=7.4$ $\mathrm{Hz}, 2 \mathrm{H}, \operatorname{ArH}), 7.39-7.44$ (m, 1H, ArH), 7.25-7.29 (m, 1H, ArH), 7.14-7.22 (m, 5H, ArH), 6.97 (d, J = 7.4 Hz, 2H, ArH), $6.77\left(\mathrm{~s}, 1 \mathrm{H}, \mathbf{H}_{\text {aminal }}\right), 6.92(\mathrm{~d}, \mathrm{~J}=6.5 \mathrm{~Hz}, 2 \mathrm{H}, \operatorname{ArH}), 4.81(\mathrm{~d}, \mathrm{~J}=8.3 \mathrm{~Hz}, 1 \mathrm{H}, \mathrm{O}-\mathrm{CH}-\mathrm{CH}-\mathrm{N}), 4.44(\mathrm{~d}, \mathrm{~J}=8.3$ $\mathrm{Hz}, 1 \mathrm{H}, \mathrm{O}-\mathrm{CH}-\mathrm{CH}-\mathrm{N}) ;{ }^{13} \mathrm{C}$ NMR $\left(500 \mathrm{MHz}, \mathrm{CDCl}_{3}\right) \delta 138.7,136.9,136.7,136.2,133.5,129.4,128.8,128.7(1)$, 128.6(6), 128.5(4), 128.4(5), 128.4(4), 128.0, 127.4, 126.6, 126.5, 91.8, 86.8, 71.5; HRMS (ESI ${ }^{+}$) calc'd for $\left[\mathrm{C}_{27} \mathrm{H}_{23} \mathrm{NO}_{3} \mathrm{SNa}\right]^{+}$requires $\mathrm{m} / z 464.1296$, found $\mathrm{m} / z$ 464.1276. Isolated as a white solid $\left(\mathrm{mp}=114-119^{\circ} \mathrm{C}\right)$. 


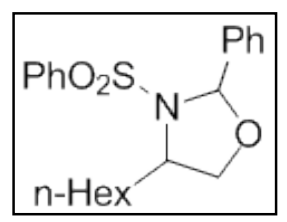

$\boldsymbol{N}$-Benzensulfonyl-4- $\boldsymbol{n}$-hexyl-2-phenyl-1,3-oxazolane. (Table 3, entry 1). Prepared according to the general procedure using $134 \mathrm{mg} 1$-octene. Because the product co-eluted with unreacted oxaziridine, the reaction mixture was treated with $110 \mathrm{mg}(1.8 \mathrm{mmol})$ dimethylsulfide for $5 \mathrm{~min}$ before dilution with hexanes and purification by flash chromatography on silica gel using 5\% EtOAc and $1 \%$ triethylamine in hexanes as eluent. Yield: $67 \mathrm{mg}(.211 \mathrm{mmol}, 15 \%) \mathrm{dr}=1.9: 1$ cis:trans. Major diastereomer $\left(2 S^{*}, 4 S^{*}\right)$ : IR (neat) 3064, 3033, 2954, 2929, 2858, 1354, 1167; ${ }^{1} \mathrm{H}$ NMR (500 MHz, CDCl $)$ d 7.88 (d, J = 7.5 Hz, 2H, $\left.-\mathrm{SO}_{2} \mathrm{PhH}_{\text {H }}\right), 7.64$ $(\mathrm{t}, \mathrm{J}=7.5 \mathrm{~Hz}, 1 \mathrm{H}, \operatorname{ArH}), 7.52-7.57(\mathrm{~m}, 4 \mathrm{H}, \operatorname{ArH}), 7.30-7.40(\mathrm{~m}, 3 \mathrm{H}, \operatorname{ArH}), 6.21\left(\mathrm{~s}, 1 \mathrm{H}, \mathbf{H}_{\text {aminal }}\right), 3.77-3.84(\mathrm{~m}, 1 \mathrm{H}$, $\left.\mathrm{N}-\mathrm{CH}-\mathrm{CH}_{2}\right), 3.70\left(\mathrm{dd}, \mathrm{J}=8.4,6.8 \mathrm{~Hz}, 1 \mathrm{H}, \mathrm{O}-\mathrm{CH}_{2}\right), 3.54\left(\mathrm{dd}, \mathrm{J}=8.4,5.3 \mathrm{~Hz}, 1 \mathrm{H}, \mathrm{O}-\mathrm{CH}_{2}\right), 1.68-1.77\left(\mathrm{~m}, 1 \mathrm{H}, \mathrm{CH}_{2}-\right.$ $\left.\mathrm{CH}_{2}-\mathrm{CH}-\mathrm{N}\right), 1.12-1.41\left(\mathrm{~m}, 9 \mathrm{H}, \mathrm{CH}_{2}\right), 0.86\left(\mathrm{t}, \mathrm{J}=7.5 \mathrm{~Hz}, \mathrm{CH}_{3}\right) ;{ }^{13} \mathrm{C} \mathrm{NMR}\left(500 \mathrm{MHz}, \mathrm{CDCl}_{3}\right): 138.7,137.9,133.4$, $129.5,128.8,128.6,128.1,126.9,91.8,70.9,60.0,35.1,31.8,29.1,26.3,22.7,14.2$; HRMS (ESI $\left.{ }^{+}\right)$calc'd for $\left[\mathrm{C}_{21} \mathrm{H}_{27} \mathrm{NO}_{3} \mathrm{SNa}\right]^{+}$requires $\mathrm{m} / \mathrm{z} 396.1609$, found $\mathrm{m} / \mathrm{z}$ 396.1593. Isolated as a colorless oil. Minor diastereomer $\left(2 R^{*}\right.$, $4 S^{*}$ ): IR (neat) 3066, 3035, 2956, 2927, 2857, 1350, 1166; ${ }^{1} \mathrm{H}$ NMR (500 MHz, CDCl $)$ : 7.40-7.49 (m, 3H, ArH), 7.14-7.34 (m, 7H, ArH), 6.11 (s, 1H, $\mathbf{H}_{\text {aminal }}$ ), 4.20 (dd, J = 8.3, $6.0 \mathrm{~Hz}, 1 \mathrm{H}, \mathrm{O}-\mathrm{CH}_{2}$ ), 4.07 (ddt, J = 9.5, 6.0, 3.6 Hz, $\left.1 \mathrm{H}, \mathrm{N}-\mathrm{CH}-\mathrm{CH}_{2}\right), 3.79\left(\mathrm{dd}, \mathrm{J}=8.3,3.6 \mathrm{~Hz}, 1 \mathrm{H}, \mathrm{O}-\mathrm{CH}_{2}\right), 2.01-2.18\left(\mathrm{~m}, 1 \mathrm{H}, \mathrm{CH}_{2}-\mathrm{CH}_{2}-\mathrm{CH}-\mathrm{N}\right), 1.65-1.83(\mathrm{~m}, 1 \mathrm{H}$, $\left.\mathrm{CH}_{2}-\mathrm{CH}_{2}-\mathrm{CH}-\mathrm{N}\right)$ 1.13-1.40 (m, 8H, $\left.\mathrm{CH}_{2}\right), 0.89\left(\mathrm{t}, \mathrm{J}=7.5, \mathrm{CH}_{3}\right) ;{ }^{13} \mathrm{C} \mathrm{NMR}\left(500 \mathrm{Mhz}, \mathrm{CDCl}_{3}\right): 141.6,137.4,132.4$, $129.2,128.8,128.3,127.9,127.1,92.0,70.7,60.2,32.6,31.9,29.4,26.2,22.8,14.3$. Isolated as a colorless oil.

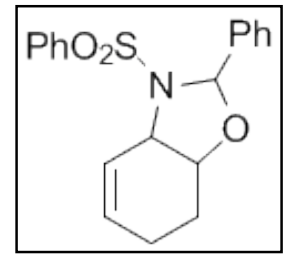

$N$-Benzensulfonyl-2-phenyl-3a,6,7,7a-tetrahydro-3H-benzooxazolane (Table 3 , entry 2 ). To a dry $25 \mathrm{~mL}$ round bottom flask containing a stir bar was added $35 \mathrm{mg}(.12 \mathrm{mmol})$ copper(II) trifluoroacetate hydrate, followed by $627 \mathrm{mg}(2.4 \mathrm{mmol})$ trans-(2-phenylsulfonyl)3-phenyloxaziridine (1) and $4.8 \mathrm{~mL}$ freshly distilled $\mathrm{CH}_{2} \mathrm{Cl}_{2}$. A dry two-dram vial was charged with $96 \mathrm{mg}(1.2 \mathrm{mmol}) 1,3$-cyclohexadiene, $42 \mu \mathrm{L}$ HMPA $(240 \mu \mathrm{mol})$ and $4 \mathrm{~mL} \mathrm{CH}_{2} \mathrm{Cl}_{2}$. The diene solution was then added via syringe to the oxaziridine solution, and the empty vial rinsed with two portions of $0.4 \mathrm{ml} \mathrm{CH} \mathrm{Cl}_{2}$. The vial was sealed with a septum, and the reaction was stirred under $\mathrm{N}_{2}$ for $1.25 \mathrm{~h}$ at $35^{\circ} \mathrm{C}$. The solvent was then removed and the mixture was directly purified on silica gel using 10\% EtOAc and 2\% triethylamine in hexanes with as eluent to give the aminal product as a mixture of two diastereomers. Yield: $298 \mathrm{mg}(.873 \mathrm{mmol}, 71.5 \%) 2.4: 1 \mathrm{dr}$. Major diastereomer $\left(2 R^{*}, 4 R^{*}, 5 S^{*}\right)$ : IR (neat) 3064 , 3033, 2922, 1382, 1353, 1284, 1261, 1239, 1213, 1169; $\left.{ }^{1} \mathrm{H} \mathrm{NMR} \mathrm{(500} \mathrm{MHz,} \mathrm{CDCl}_{3}\right) \delta 7.76(\mathrm{~d}, \mathrm{~J}=8.2 \mathrm{~Hz} 2 \mathrm{H}, \mathrm{ArH})$, $7.57(\mathrm{t}, \mathrm{J}=7.7 \mathrm{~Hz}, 1 \mathrm{H}, \operatorname{ArH}), 7.48(\mathrm{t}, \mathrm{J}=7.7 \mathrm{~Hz}, 2 \mathrm{H}, \operatorname{ArH}), 7.42-7.45(\mathrm{~m}, 2 \mathrm{H}, \operatorname{ArH}), 7.30-7.32(\mathrm{~m}, 2 \mathrm{H}, \operatorname{ArH}), 5.94$ $\left(\mathrm{s}, 1 \mathrm{H}, \mathbf{H}_{\text {aminal }}\right), 5.89(\mathrm{dd}, 5.8,10 \mathrm{~Hz}, 1 \mathrm{H}, \mathrm{CH}=\mathrm{CHCN}), 5.61(\mathrm{~d}, \mathrm{~J}=10 \mathrm{~Hz}, 1 \mathrm{H}, \mathrm{CH}=\mathrm{CHCN}), 4.43(\mathrm{~m}, 1 \mathrm{H}$, CHNCHO), $3.90(\mathrm{~m}, 1 \mathrm{H}, \mathrm{CHNCHO}), 2.12-2.21\left(\mathrm{~m}, 1 \mathrm{H}, \mathrm{CH}_{2} \mathrm{CHO}\right), 2.05(\mathrm{dt}, \mathrm{J}=15,4.3 \mathrm{~Hz}, 1 \mathrm{H}, \mathrm{CH} 2) 1.92(\mathrm{dt}, \mathrm{J}=$ $\left.17.7,5.1 \mathrm{~Hz}, 1 \mathrm{H}, \mathrm{C}=\mathrm{CCH}_{2}\right), 1.66-1.73\left(\mathrm{~m}, 1 \mathrm{H}, \mathrm{CH}_{2}\right) ;{ }^{13} \mathrm{C} \mathrm{NMR}(500 \mathrm{MHz}, \mathrm{CDCl} 3) \delta 138.7,138.4,133.0,129.7$, 129.1, 129.0, 128.2, 127.6, 127.6, 125.0, 91.7, 75.2, 56.4, 24.3, 18.8; HRMS $\left(\mathrm{ESI}^{+}\right)$calc'd for $\left[\mathrm{C}_{19} \mathrm{H}_{19} \mathrm{NO}_{3} \mathrm{SNa}^{+}\right.$ requires $\mathrm{m} / \mathrm{z} 364.0983$, found $\mathrm{m} / \mathrm{z} 364.0984$. Isolated as a clear soild $\left(\mathrm{mp}=124-126{ }^{\circ} \mathrm{C}\right)$. Minor diastereomer $\left(2 S^{*}, 4 R^{*}, 5 S^{*}\right)$; IR (neat) $3057,2925,1344,1266,1215,1160 ;{ }^{1} \mathrm{H}$ NMR $\left(500 \mathrm{MHz}, \mathrm{CDCl}_{3}\right) \delta 7.46(\mathrm{~d}, \mathrm{~J}=7.4 \mathrm{~Hz}$, $2 \mathrm{H}, \operatorname{ArH}), 7.40(\mathrm{t}, \mathrm{J}=7.4 \mathrm{~Hz}, 1 \mathrm{H}, \operatorname{ArH}), 7.21-7.31(\mathrm{~m}, 5 \mathrm{H}, \operatorname{ArH}), 7.16(\mathrm{t}, \mathrm{J}=7.4 \mathrm{~Hz}, 1 \mathrm{H}, \operatorname{ArH}), 6.11(\mathrm{~d}, \mathrm{~J}=10.4$ $\mathrm{Hz}, 1 \mathrm{H}, \mathrm{CH}=\mathrm{CHCN}), 6.01-6.04(\mathrm{~m}, 1 \mathrm{H}, \mathrm{CH}=\mathrm{CHCN}), 6.00\left(\mathrm{~s}, 1 \mathrm{H}, \mathbf{H}_{\text {aminal }}\right), 4.59-4.62(\mathrm{~m}, 1 \mathrm{H}, \mathrm{CHNCHO}), 4.55-$ $4.58(\mathrm{~m}, 1 \mathrm{H}, \mathrm{CHNCHO}), 2.13-2.21\left(\mathrm{~m}, 1 \mathrm{H}, \mathrm{CH}_{2}\right), 1.90-1.97\left(\mathrm{~m}, 1 \mathrm{H}, \mathrm{C}=\mathrm{CCH}_{2}\right), 1.81-1.87\left(\mathrm{~m}, 1 \mathrm{H}, \mathrm{C}=\mathrm{CC} \mathbf{H}_{2}\right)$, $1.68-1.75\left(\mathrm{~m}, 1 \mathrm{H}, \mathrm{CH}_{2}\right) ;{ }^{13} \mathrm{C} \mathrm{NMR}(500 \mathrm{MHz}, \mathrm{CDCl} 3) \delta 141.1,137.5,132.2,132.0,129.0,128.4,128.1,127.8$, 127.2, 123.3, 90.4, 74.9, 57.3, 24.8, 19.6 ; HRMS $\left(\mathrm{ESI}^{+}\right)$calc'd for $\left[\mathrm{C}_{19} \mathrm{H}_{19} \mathrm{NO}_{3} \mathrm{SNa}\right]^{+}$requires $m / z 364.0983$, found $\mathrm{m} / \mathrm{z}$ 363.0989. Isolated as a white solid $\left(\mathrm{mp}=70-74^{\circ} \mathrm{C}\right)$.

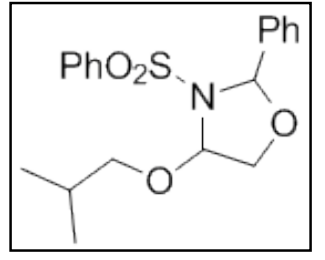

$N$-Benzenesulfonyl-3-isobutoxy-2-phenyl-1,3-oxazolane (Table 3, entry 3 To a dry $25 \mathrm{~mL}$ round bottom flask containing a stir bas was added $35 \mathrm{mg}$ (.12 mmol) copper(II) trifluoroacetate hydrate, followed by $627 \mathrm{mg}(2.4 \mathrm{mmol})$ trans-(2-phenylsulfonyl)-3phenyloxaziridine (1) and $4.8 \mathrm{~mL}$ freshly distilled $\mathrm{CH}_{2} \mathrm{Cl}_{2}$. A dry two-dram vial was charged with $120 \mathrm{mg}(1.2 \mathrm{mmol})$ isobutyl vinyl ether, $42 \mu \mathrm{L}$ HMPA $(240 \mu \mathrm{mol})$ and $4 \mathrm{~mL}$ $\mathrm{CH}_{2} \mathrm{Cl}_{2}$. The vinyl ether solution was then added via syringe to the oxaziridine solution, and

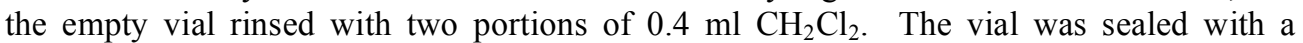
septum, and the reaction was stirred under $\mathrm{N}_{2}$ for $0.5 \mathrm{~h}$ at $35^{\circ} \mathrm{C}$. The solvent was then removed and the mixture was directly purified on silica gel using 10\% EtOAc and 2\% triethylamine in hexanes with as eluent to give the aminal product as a mixture of two diastereomers. Yield: $306 \mathrm{mg}(.847 \mathrm{mmol}, 70.6 \%), 1: 1.4$ cis:trans. Major diastereomer $\left(2 S^{*}, 4 R^{*}\right)$ : IR (neat) $3066,3035,2958,2929,2874,1353,1167 ;{ }^{1} \mathrm{H}$ NMR $\left(500 \mathrm{MHz}, \mathrm{CDCl}_{3}\right) \delta 7.77$ $\left(\mathrm{d}, \mathrm{J}=7.2 \mathrm{~Hz}, 2 \mathrm{H},-\mathrm{SO}_{2} \mathrm{PhH}\right), 7.58(\mathrm{t}, \mathrm{J}=7.6 \mathrm{~Hz}, 1 \mathrm{H}, \mathrm{ArH}), 7.45-7.50,(\mathrm{~m}, 4 \mathrm{H}, \mathrm{ArH}), 7.28-7.33(\mathrm{~m}, 3 \mathrm{H}, \operatorname{ArH})$, $6.12\left(\mathrm{~s}, 1 \mathrm{H}, \mathbf{H}_{\text {aminal }}\right), 4.38\left(\mathrm{dd}, \mathrm{J}=4.7,1.7 \mathrm{~Hz}, 1 \mathrm{H},-\mathrm{OCHCH}_{2}\right) 3.98\left(\mathrm{dd}, \mathrm{J}=9.3,1.7 \mathrm{~Hz}, 1 \mathrm{H},-\mathrm{OCHCH}_{2}\right), 3.62(\mathrm{dd}, \mathrm{J}$ $\left.=9.3,4.7 \mathrm{~Hz}, 1 \mathrm{H},-\mathrm{OCHCH}_{2}\right), 3.49\left(\mathrm{dd}, \mathrm{J}=9.2,6.2 \mathrm{~Hz}, 1 \mathrm{H},-\mathrm{OCH}_{2} \mathrm{CH}(\mathrm{Me})_{2}\right), 3.26(\mathrm{dd}, \mathrm{J}=9.2,6.4 \mathrm{~Hz}, 1 \mathrm{H},-$ 
$\left.\mathrm{OCH}_{2} \mathrm{CH}(\mathrm{Me})_{2}\right), 1.77$ (m, 1H, -CH(Me $\left.)_{2}\right) ;{ }^{13} \mathrm{C}$ NMR (500 MHz, CDCl3) 139.0, 138.1, 133.4, 129.4, 129.0, 128.4, 127.7, 127.4, 92.5, 89.2, 74.9, 72.6, 28.4, 19.4(9), 19.4(8); HRMS $\left(\mathrm{ESI}^{+}\right)$calc'd for $\left[\mathrm{C}_{19} \mathrm{H}_{23} \mathrm{NO}_{4} \mathrm{SNa}^{+}\right.$requires $\mathrm{m} / z$ 384.1245 , found $m / z 384.1260$. Isolated as a clear soild $\left(\mathrm{mp}=87-88^{\circ} \mathrm{C}\right)$. Minor diastereomer $\left(2 S^{*}, 4 S^{*}\right)$ : IR (neat) 3065, 3035, 2958, 2931, 2873, 1357, 1171; ${ }^{1} \mathrm{H}$ NMR (500 MHz, $\left.\mathrm{CDCl}_{3}\right) \delta 7.64$ (d, J = 7.2 Hz, 2H, -SO $\left.\mathrm{Sh}_{2} \mathrm{Ph}\right), 7.48$ $(\mathrm{t}, \mathrm{J}=7.4 \mathrm{~Hz}, 1 \mathrm{H}, \operatorname{ArH}), 7.33-7.38,(\mathrm{~m}, 2 \mathrm{H}, \operatorname{ArH}), 7.27-7.32(\mathrm{~m}, 3 \mathrm{H}, \operatorname{ArH}), 7.22-7.27(\mathrm{~m}, 2 \mathrm{H}, \operatorname{ArH}), 6.02(\mathrm{~s}, 1 \mathrm{H}$, $\left.\mathbf{H}_{\text {aminal }}\right), 5.58\left(\mathrm{~d}, \mathrm{~J}=3.6 \mathrm{~Hz}, 1 \mathrm{H},-\mathrm{OCHCH}_{2}\right) 4.11(\mathrm{dd}, \mathrm{J}=9.5,3.6 \mathrm{~Hz}, 1 \mathrm{H},-\mathrm{OCHCH})_{2}, 3.99(\mathrm{~d}, \mathrm{~J}=9.5 \mathrm{~Hz}, 1 \mathrm{H},-$ $\left.\mathrm{OCHCH}_{2}\right), 3.53\left(\mathrm{dd}, \mathrm{J}=9.0,6.7 \mathrm{~Hz}, 1 \mathrm{H},-\mathrm{OCH}_{2} \mathrm{CH}(\mathrm{Me})_{2}\right), 3.22\left(\mathrm{dd}, \mathrm{J}=9.0,6.7 \mathrm{~Hz}, 1 \mathrm{H},-\mathrm{OCH}_{2} \mathrm{CH}(\mathrm{Me})_{2}\right), 1.80(\mathrm{~m}$, $\left.1 \mathrm{H},-\mathrm{CH}(\mathrm{Me})_{2}\right), 0.91\left(\mathrm{~d}, \mathrm{~J}=6.7 \mathrm{~Hz}, 3 \mathrm{H}, \mathrm{CH}_{3}\right), 0.86\left(\mathrm{~d}, \mathrm{~J}=6.7 \mathrm{~Hz}, 3 \mathrm{H}, \mathrm{CH}_{3}\right) ;{ }^{13} \mathrm{C}$ NMR $(500 \mathrm{MHz}, \mathrm{CDCl} 3) 139.0$, 138.1, 133.4, 129.4, 129.0, 128.4, 127.7, 127.4, 92.5, 89.2, 74.9, 72.6, 28.4, 19.4(9), 19.4(8); HRMS (ESI') calc'd for $\left[\mathrm{C}_{19} \mathrm{H}_{23} \mathrm{NO}_{4} \mathrm{SNa}\right]^{+}$requires $m / z 384.1245$, found $m / z 384.1261$. Isolated as a colorless oil.

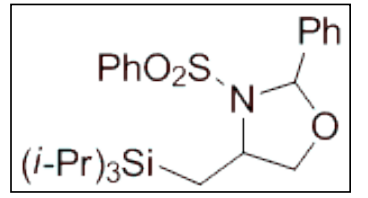

$N$-Benzensulfonyl-2-phenyl-4-[(triisopropylsilyl)-methyl]-1,3-oxazolane (Table 3 , entry 4). Prepared according to the general procedure using $237.8 \mathrm{mg}$ allyltriisopropylsilane and purified on silica gel with $10 \%$ ethyl acetate and 3\% triethylamine in hexanes. Yield: $362 \mathrm{mg}$ (.788 mmol, 65.7\%), 1.2:1 cis:trans. Major diastereomer $\left(2 S^{*}, 4 R^{*}\right)$ : IR (neat) $3065,3033,2943,2890,2866,1354,1167 ;{ }^{1} \mathrm{H}$ NMR $\left(500 \mathrm{MHz}, \mathrm{CDCl}_{3}\right) \delta$ 7.85-7.89 (m, 2H, ArH), 7.52-7.65 (m, 5H, ArH), 7.33-7.41, (m, 3H, ArH), 6.21 (s, 1H, $\mathbf{H}_{\text {aminal }}$ ), $4.04\left(\mathrm{dtd}, \mathrm{J}=12.6,6.6,2.4 \mathrm{~Hz}, 1 \mathrm{H}, \mathrm{OCH}_{2} \mathrm{CHN}\right), 3.79\left(\mathrm{dd}, \mathrm{J}=8.8,6.6 \mathrm{~Hz}, 1 \mathrm{H}, \mathrm{OCH}_{2} \mathrm{CHN}\right), 3.45(\mathrm{dd}, \mathrm{J}=$ 8.8, 6.6 Hz, 1H, OCH $\left.\mathbf{H}_{2} \mathrm{CHN}\right), 1.53$ (dd, J = 12.6, $\left.2.4 \mathrm{~Hz}, 1 \mathrm{H}, \mathrm{SiCH}_{2} \mathrm{CHN}\right), .04-1.05$ (m, 21H, $i$-Pr-H), 0.88 (dd, J = 12.6, $\left.12.6 \mathrm{~Hz}, 1 \mathrm{H}, \mathrm{SiCH}_{2} \mathrm{CHN}\right) ;{ }^{13} \mathrm{C}$ NMR $(500 \mathrm{MHz}, \mathrm{CDCl} 3)$ 138.7, 137.9, 133.4, 129.4, 128.9, 128.6, 128.1, 127.1, 91.6, 72.1, 58.6, 18.8, 18.8(5), 17.2, 11.4; HRMS $\left(\mathrm{ESI}^{+}\right)$calc'd for $\left[\mathrm{C}_{25} \mathrm{H}_{37} \mathrm{NO}_{3} \mathrm{SSiNa}^{+}\right.$requires $\mathrm{m} / \mathrm{z}$ 482.2161, found $m / z 482.2160$. Isolated as a white solid $\left(\mathrm{mp}=85-89{ }^{\circ} \mathrm{C}\right)$. Minor diastereomer $\left(2 R^{*}, 4 R^{*}\right)$; IR (neat) 3066, 2942, 2890, 2866, 1348, 1162; ${ }^{1} \mathrm{H}$ NMR $\left(500 \mathrm{MHz}, \mathrm{CDCl}_{3}\right) \delta 7.53-7.57$ (m, 2H, ArH), 7.45 (tt, J = 7.6, 1.4 $\mathrm{Hz}, 1 \mathrm{H}, \operatorname{ArH}), 7.26-7.34(\mathrm{~m}, 5 \mathrm{H}, \operatorname{ArH}), 7.21-7.25(\mathrm{~m}, 2 \mathrm{H}, \operatorname{ArH}), 6.22\left(\mathrm{~s}, 1 \mathrm{H}, \mathbf{H}_{\text {aminal }}\right), 4.33-4.39(\mathrm{~m}, 1 \mathrm{H}$, $\left.\mathrm{OCH}_{2} \mathrm{CHN}\right), 4.18\left(\mathrm{ddd}, \mathrm{J}=8.2,5.8, .9 \mathrm{~Hz}, 1 \mathrm{H}, \mathrm{OCH}_{2} \mathrm{CHN}\right), 3.64\left(\mathrm{dd}, \mathrm{J}=8.2,4.1 \mathrm{~Hz}, 1 \mathrm{H}, \mathrm{OCH}_{2} \mathrm{CHN}\right), 1.64$ (ddd, $\mathrm{J}$ $\left.=14.3,2.3,0.9 \mathrm{~Hz}, 1 \mathrm{H}, \mathrm{SiCH}_{2} \mathrm{CHN}\right), 1.00-1.11\left(\mathrm{~m}, 22 \mathrm{H}, \mathrm{SiCH}_{2} \mathrm{CHN}\right.$ and $\left.\operatorname{Si}\left(i-\mathrm{Pr}_{3}\right)\right) ;{ }^{13} \mathrm{C} \operatorname{NMR}\left(500 \mathrm{MHz}, \mathrm{CDCl}_{3}\right) \delta$ 142.2, 137.9, 132.4, 129.1, 128.8, 128.3, 127.7, 127.2, 91.1, 72.2, 58.8, 19.0, 18.9, 13.9, 11.5; HRMS (ESI ${ }^{+}$) calc'd for $\left[\mathrm{C}_{25} \mathrm{H}_{37} \mathrm{NO}_{3} \mathrm{SSiNa}\right]^{+}$requires $m / z 482.2161$, found $\mathrm{m} / z$ 482.2172. Isolated as a colorless oil.

\section{Hydrolysis of the benzylidene aminal.}

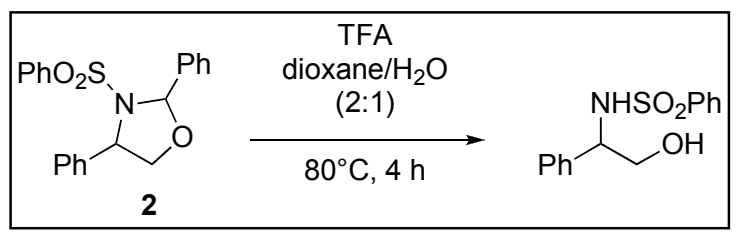

$N$-Benzenesulfonyl-2-amino-2-phenylethanol.

This procedure is a modification of that developed by Langlois and coworkers. ${ }^{3}$ To a dry 2 dram vial containing a stir bar was added $170 \mathrm{mg}(.47 \mathrm{mmol})$ of $N$-benzensulfonyl-2,4-diphenyl1,3-oxazolane 2 , followed by $2 \mathrm{ml} \mathrm{1,4-dioxane/water} \mathrm{(2:1).}$ Trifluoroacetic acid $(70 \mu \mathrm{L}, .93 \mathrm{mmol})$ was then added dropwise to the stirred solution. The vial was subsequently flushed with nitrogen, sealed with a Teflon cap, and heated to $80{ }^{\circ} \mathrm{C}$ in an oil bath for $4 \mathrm{~h}$. The mixture was then diluted with EtOAc $(5 \mathrm{ml})$ and poured onto $10 \mathrm{ml}$ of aqueous saturated sodium bicarbonate. The organic layer was separated and washed with $10 \mathrm{ml}$ brine before removal of the solvent under reduced pressure. The resulting product was purified on silica gel with 1:1 EtOAc:hexanes as eluent to give the $N$-sulfonyl amino alcohol (123 mg, $.442 \mathrm{mmol}, 95 \%$ yield). IR (neat) 3489 , 3277, 3064, 3032, 2918, 2879, 1322, 1158; ${ }^{1} \mathrm{H}$ NMR $\left(500 \mathrm{MHz}, \mathrm{CDCl}_{3}\right) \delta 7.71(\mathrm{~m}, 2 \mathrm{H},-\mathrm{SO} 2 \mathrm{PhH}), 7.45(\mathrm{tt}, \mathrm{J}=7.4$, $1.3 \mathrm{~Hz}, 1 \mathrm{H}, \operatorname{ArH}), 7.30-7.38,(\mathrm{~m}, 2 \mathrm{H}, \mathrm{ArH}), 7.13-7.19$ (m, 3H, ArH), 7.04-7.11 (m, 2H, ArH), 5.87 (d, J = 7.2 Hz, $1 \mathrm{H}, \mathrm{NH}), 4.44-4.52\left(\mathrm{~m}, 1 \mathrm{H}, \mathrm{NHCHCH}_{2}\right), 3.70-3.80\left(\mathrm{~m}, 2 \mathrm{H}, \mathrm{OCH}_{2} \mathrm{CHN}\right), 2.53$ (bs, $\left.1 \mathrm{H}, \mathrm{OH}\right) ;{ }^{13} \mathrm{C} \mathrm{NMR}(500 \mathrm{MHz}$, CDCl3) 140.3, 137.5, 132.7, 129.0, 128.8, 128.1, 127.3, 127.1, 66.4, 59.9; HRMS (ESI') calc'd for $\left[\mathrm{C}_{14} \mathrm{H}_{15} \mathrm{NO}_{3} \mathrm{~S}-\mathrm{H}\right]^{-}$ $\left.([\mathrm{M}-\mathrm{H}]]^{-}\right)$requires $\mathrm{m} / \mathrm{z} 276.0695$, found $\mathrm{m} / \mathrm{z} 276.0695$. Isolated as a white solid $\left(\mathrm{mp}=104.5-105^{\circ} \mathrm{C}\right)$.

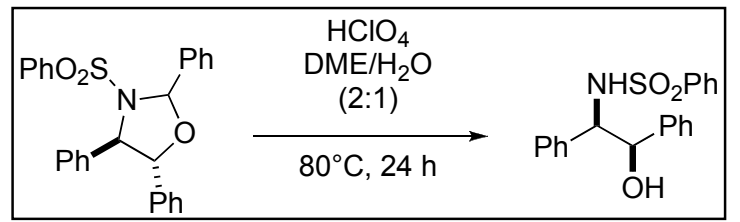

$\left(1 R^{*}, \quad 2 R *\right)-2-N$-benzenesulfonamide-1,2-diphenylethanol. To a dry 2 dram vial containing a stir bar was added $100 \mathrm{mg}$ (.226 $\mathrm{mmol})$ of $N$-benzensulfonyl-2,4,5-triphenyl-1,3oxazolane, 1.9:1 mixture of 3 and 4 , followed by $2.8 \mathrm{ml} \mathrm{1,2-}$ dimethoxyethane/water (2:1). Perchloric acid $(170 \mu \mathrm{L}, 2.8$ mmol) was then added dropwise to the stirred solution. The vial was subsequently flushed with nitrogen, sealed with a Teflon cap, and heated to $80{ }^{\circ} \mathrm{C}$ in an oil bath for $24 \mathrm{~h}$. The mixture was then diluted with EtOAc $(5 \mathrm{ml})$ and poured onto $10 \mathrm{ml}$ of saturated aqueous $\mathrm{NaHCO}_{3}$. The organic layer was separated and washed with $10 \mathrm{ml}$ brine and dried with $\mathrm{Na}_{2} \mathrm{SO}_{4}$ before removal of the solvent under 
reduced pressure. The resulting product was purified on silica gel with 1:1 EtOAc:hexanes as eluent to give the syn$N$-sulfonylamino alcohol (64.1 mg, .182 mmol, 80\% yield) as a single diastereomer. IR (neat) 3490, 3286, 3063, 3032, 2921, 1324, 1160; ${ }^{1} \mathrm{H}$ NMR $\left(500 \mathrm{MHz}, \mathrm{CDCl}_{3}\right) \delta 8.08\left(\mathrm{~d}, \mathrm{~J}=8.9 \mathrm{~Hz}, 1 \mathrm{H}, \mathrm{NH}-\mathrm{SO}_{2} \mathrm{Ph}\right), 7.44(\mathrm{~d}, \mathrm{~J}=7.4 \mathrm{~Hz}$, $\left.2 \mathrm{H},-\mathrm{SO}_{2} \mathrm{PhH}\right), 7.36\left(\mathrm{t}, \mathrm{J}=7.4 \mathrm{~Hz}, 1 \mathrm{H},-\mathrm{SO}_{2} \mathrm{PhH}\right), 7.24\left(\mathrm{t}, \mathrm{J}=7.4 \mathrm{~Hz}, 2 \mathrm{H},-\mathrm{SO}_{2} \mathrm{PhH}\right), 7.17-7.13(\mathrm{~m}, 5 \mathrm{H}, \mathrm{ArH})$, $7.05-7.98(\mathrm{~m}, 5 \mathrm{H}, \mathrm{ArH}), 5.51(\mathrm{~d}, \mathrm{~J}=4.5 \mathrm{~Hz}, 1 \mathrm{H}, \mathrm{OH}), 4.66(\mathrm{t}, \mathrm{J}=5.0 \mathrm{~Hz}, 1 \mathrm{H}, \mathrm{CHOH}), 4.45$ (dd, J = 8.9 Hz, 5.0 Hz, $1 \mathrm{H}, \mathrm{CHNH}) ;{ }^{13} \mathrm{C}$ NMR (500 MHz, DMSO-d 6 ) $\delta$ 142.3, 141.6, 139.5, 131.4, 128.3, 127.6, 127.5, 127.3, 126.9, 126.8, 126.4, 126.1, 75.9, 64.0; HRMS (ESI') calc'd for $\left[\mathrm{C}_{20} \mathrm{H}_{19} \mathrm{NO}_{3} \mathrm{SNa}\right]^{+}$requires $\mathrm{m} / \mathrm{z} 376.0983$, found $\mathrm{m} / \mathrm{z}$ 376.0996. Isolated as a white solid $\left(\mathrm{mp}=144-145^{\circ} \mathrm{C}\right)$. The complementary anti $\left(1 \mathrm{R}^{*}, 2 \mathrm{~S}^{*}\right)$ diastereomer has previously been reported ${ }^{4}$ and is spectroscopically distinguishable from the syn isomer.

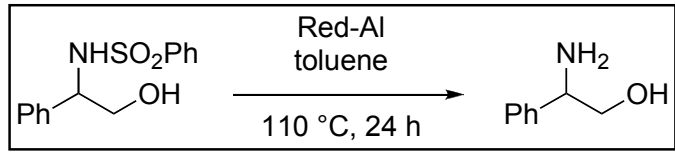

$\mathrm{mL}(10.1 \mathrm{mmol})$ of $65 \%$ wt. sodium bis(2-methoxyethoxy)aluminum hydride solution in toluene was added dropwise. The solution was refluxed for 24 hours before being cooled to room temperature and quenched with 25 $\mathrm{mL}$ of $10 \% \mathrm{NaOH}$. The biphasic mixture was extracted with $50 \mathrm{~mL}$ of dichloromethane. The organic extract was then washed with $10 \% \mathrm{NaOH}$ and brine before being dried with $\mathrm{Na}_{2} \mathrm{SO}_{4}$ and concentrated. The product was purified on silica gel using a $4 \%$ solution of $\mathrm{MeOH}$ in ammonio-saturated $\mathrm{CH}_{2} \mathrm{Cl}_{2}$ to give $112.8 \mathrm{mg}(.823 \mathrm{mmol}, 33 \%$ yield) of 2-phenylglycinol. All spectral data were in complete agreement with those previously reported for 2phenylglycinol. ${ }^{5}$

\section{References:}

${ }^{1}$ a) Vishwakarma, L. C.; Stringer, O. D.; Davis, F. A.; checked by Pribish, J.; Vedejs, E. Org. Syn. 1993, 8, 546; Org. Syn. 1988, 66, 203. b) Davis, F. A.; Chattopadhyay, S.; Towson, J. C.; Lal, S.; Reddy, T. J. Org. Chem. 1988, $53,2087$.

${ }^{2}$ Synthesized according to procedure by Foote, et al. Foote, C. S.; Denny, R. W. J. Am. Chem. Soc. $1971,93,5162$.

${ }^{3}$ Langolis, N.; Nguyen, B. K. L.; Retailleau, P.; Tarnus, C.; Salomon, E. Tetrahedron Asymm. 2006, $17,53$.

${ }^{4}$ Cho, B. T.; Chun, Y. S. Synth. Commun. 1999, 29, 521.

${ }^{5}$ Gold, E. H.; Babad, E. J. Org. Chem. 1972, 37, 2208.

${ }^{6}$ Periasamy, M.; Sivakumar, S.; Redd, M. N. Synthesis 2003, 13, 1965. 


\section{NOE correlations}

NOE correlations were used to determine the relative stereochemistry of each diastereomer in Table 2-3, and the correlations observed for the major isomer are provided below. NOE correlation data for both diastereomers of the products shown in Scheme 1 are also provided.

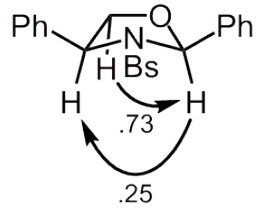

Table 2, entry 1

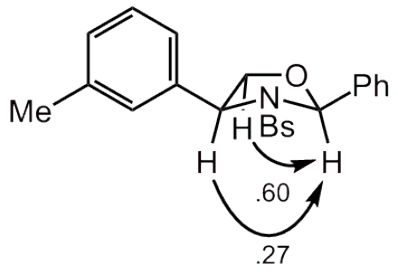

Table 2, entry 5

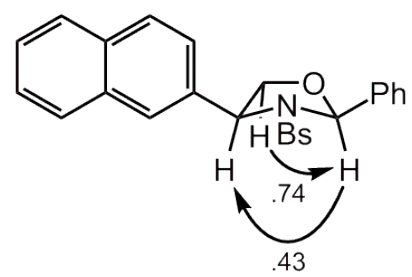

Table 2, entry 9

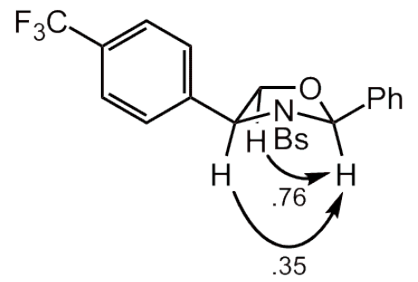

Table 2, entry 2

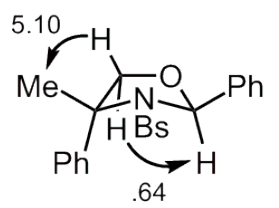

Table 2, entry 6

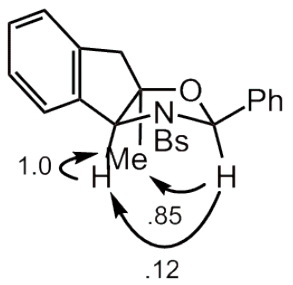

Table 2, entry 10

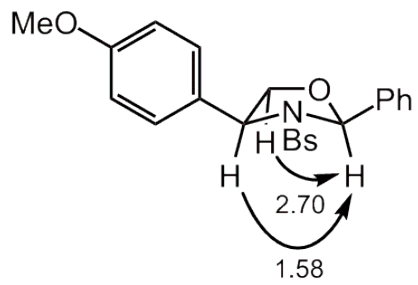

Table 2, entry 3

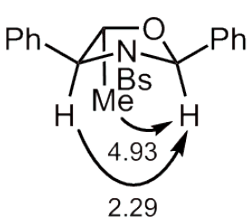

Table 2, Entry 7

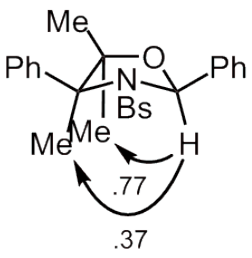

Table 2, entry 11

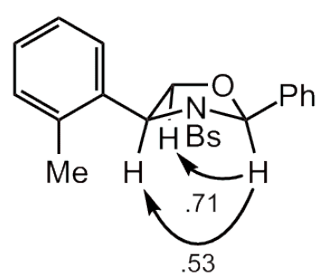

Table 2 , entry 4

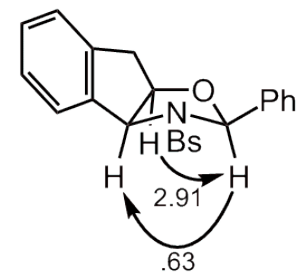

Table 2, Entry 8

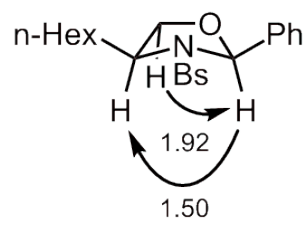

Table 3, Entry 1

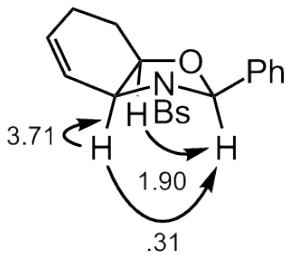

Table 2, entry 2

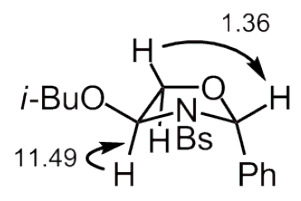

Table 2, entry 3

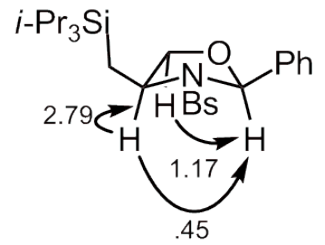

Table 2, entry 4

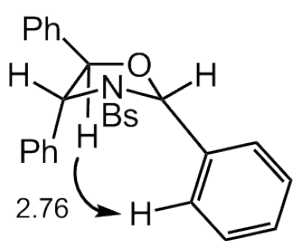

Minor diastereomer

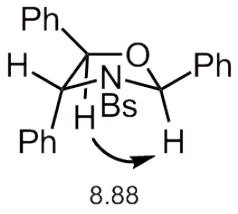

Major diastereomer

Scheme 1 